\title{
Promontofixation cœlioscopique : les points clefs de la technique chirurgicale
}

\section{Laparoscopic sacrocolpopexy: surgical key points}

Laurent Wagnera, Adrien Vidart ${ }^{\mathrm{b}}$, Caroline Thuillier ${ }^{\mathrm{c}}$

aService d'Urologie-Andrologie, CHU de NIMES, Groupe Hospitalo-Universitaire Carremeau, Place du professeur Debré, 30065 NIMES Cedex, France

bService d'urologie, Hôpital Foch, 40 rue Worth, 92150 Suresnes, France

cService d'urologie, CHU Grenoble Alpes, 38700 La Tronche, France

Auteur correspondant :

Laurent WAGNER

Tel : +33.4.66.68.33.51

Fax : +33.4 .66 .68 .37 .20$

laurent.wagner@chu-nimes.fr 


\section{Résumé}

Introduction et Objectifs : La promontofixation cœlioscopique est considérée comme le traitement de choix du prolapsus de la femme jeune car elle associe les avantages de la chirurgie prothétique par voie abdominale, avec celles d'une technique mini-invasive. De nombreuses variantes se sont développées autour de cette intervention et rendent parfois difficile l'analyse des résultats. L'objectif de cet article est de faire une mise au point sur les différents aspects de la technique chirurgicale.

Méthode : Description et recommandations sur les différents aspects de la technique chirurgicale fondée l'expérience des auteurs (trucs et astuces) mais aussi à partir des données de la littérature.

Résultats: Nous décrirons les différents temps de la double promontofixation cœlioscopique avec conservation utérine. Bien qu'il n'existe pas de données comparatives concernant les sites et modes de fixation des prothèses, il convient de se référer aux résultats des grandes séries et de privilégier de la fixation des prothèses à l'aide de fils, en avant sur l'isthme utérin et la paroi vaginale antérieure et en arrière sur les muscles releveurs. De même il est conseillé de fixer sans tension excessive les prothèses sur le promontoire à l'aide de fils non résorbables et d'effectuer une péritonisation de ces prothèses. IL n'est pas recommandé de mettre systématiquement une prothèse postérieure inter recto-vaginale en prévention de rectocèle secondaire.

\section{Conclusion}

L'utilisation de prothèses non résorbables par voie haute (promontofixation) permet de traiter les prolapsus des organes pelviens avec des bons résultats et peu de complications y compris en termes d'exposition et d'infection de prothèses. Elle est considérée à ce titre comme la technique chirurgicale de référence et les différents aspects de la technique chirurgicale doivent donc à ce titre être bien codifiés.

Mots-clés : Techniques chirurgicales; cystocèle; prolapsus utérin; rectocèle ; prothèses ; recommandations ; 


\section{Summary}

Introduction and Objectives: Laparoscopic sacrocolpopexy is considered as the gold standard for the surgical treatment for urogenital prolapse because it combines the advantages of abdominal route for prosthetic prolapse repair with those of a minimally invasive technique. Many variants have been described and make results difficult to analyze. The purpose of this article is to focus on the differents technical aspects of this surgery.

Method: Description and recommendations on different aspects of the surgical technique based on the experience of the authors (tips and tricks) but also from the data of the literature.

Results: We will describe the different steps of laparoscopic sacrocolpopexy with two mesh and with uterine preservation. Although there are no comparative data on sites and methods of fixation of prostheses, we have to refer to the results of large series. There is a consensus on fixation of prostheses with the help of threads, forward on uterine isthmus and the anterior vaginal wall and back on the levator muscles. Similarly consensus is to fix prostheses on the promontory without excessive tension and with non-absorbable thread and close the peritoneum on these prostheses. It is not recommended to systematically put an inter-recto-vaginal prosthesis in prevention of secondary rectocele.

Conclusion: Laparoscopic sacrocolpopexy with non-resorbable prostheses can treat pelvic organ prolapse with good results and few complications including in terms of prothetic exposure or infection. Considered as the reference surgical technique for prolapse repair, the various aspects of this procedure must therefore be well codified.

Keywords: Surgical technique; cystocele; uterine prolapse; rectocele; mesh; recommendations 


\section{INTRODUCTION}

Bien que le principe de base de la promontofixation soit représenté par la suspension de l'utérus sur le ligament vertébral antérieur du promontoire à l'aide de matériel prothétique, de nombreuses variantes techniques se sont développées. Le chirurgien doit cependant respecter certaines règles validées par les grandes séries de la littérature [1, 2, 3]. Aujourd'hui sont regroupées sous ce terme plusieurs variantes par: - La profondeur ou la largeur de la dissection. Notamment au niveau recto-vaginal, celleci pouvant descendre jusqu'aux muscles élévateurs de l'anus ou simplement jusqu'à avoir une surface libre sur le vagin pour poser la prothèse ;

- La fixation ou non de la prothèse dans les espaces de dissection et le mode de fixation : certaines équipes proposent de ne pas fixer la prothèse au vagin, d'autres mettent systématiquement des points d'amarrages; La fixation par fils est remplacée par certains par d'autres moyens (agrafes ou tackers).

- La mise en place d'une seule prothèse sous-vésicale fixée au niveau de la paroi vaginale antérieure et de l'isthme utérin, s'il n'y a pas de prolapsus de l'étage postérieur pour certains ou pour d'autres, de 2 prothèses systématiques, l'une sous vésicale et l'autre pré rectale [4].

\section{TECHNIQUE DE DOUBLE PROMONTOFIXATION COELIOSCOPIQUE}

\section{Installation de l'opéré}

La patiente est installée en décubitus dorsal, position gynécologique avec les jambes légèrement fléchies. Il est préférable que la patiente soit directement au contact du gel de la table (à condition que ce dernier soit bien sec) car l'interposition d'un drap peut favoriser le glissement progressif de la patiente lorsque la table sera inclinée en Trendelenburg. Un coussin triangulaire positionné sous la tête de la patiente, au contact des épaules aidera également à éviter ce glissement. Il faut s'assurer que les fesses soient bien au ras de la table afin de faciliter la mobilisation de la valve vaginale. Les deux bras sont installés le long du corps et maintenues par des gouttières. Il faut s'assurer qu'il n'y a pas de points de compression, en particulier au niveau des reliefs 
osseux articulaires (épaule, coude, poignet) et que bras, avant bras et main reposent sans contraintes sur un gel (fig. 1).

\section{Mise en place des trocarts}

Une « open cœlioscopie » ombilicale doit être la règle car elle permet d'éviter des complications vasculaires dramatiques comme une plaie aorto-iliaque (axe vasculaire dans l'axe du trocart et faible distance lors de la dépression pariétale. Une traction avec 2 pinces sur le versant interne de l'ombilic permettra de faire une incision arciforme qui sera plus discrète car secondairement enfouie une fois la traction relâchée (fig. 2). Après ouverture de l'aponévrose et du péritoine pariétal antérieur, il est bon de vérifier systématiquement au doigt l'absence d'accolement digestif au niveau du site d'introduction du trocart afin d'éviter une plaie intestinale qui pourrait passer inaperçue. Une position de Trendelenburg marquée $\left(30^{\circ}\right)$ est effectuée avant de démarrer l'insufflation par le trocart ombilical permettant à l'intestin grêle de réintégrer la cavité abdominale et libère ainsi la cavité pelvienne. Trois autres trocarts sont placés sous contrôle de la vue (optique $0^{\circ}$ sur le trocart ombilical) : 2 trocarts de $5 \mathrm{~mm}$ de diamètre sont placés en fosses iliaques droite et gauche (à 2 travers de doigt au dessus et en dedans de des épines iliaques antéro-supérieures) et 1 trocart de 5 (ou $10 \mathrm{~mm}$ ) à midistance entre ombilic et symphyse pubienne.

\section{Exposition}

L'exposition de la cavité pelvienne est un temps extrêmement important car conditionne le reste de la procédure chirurgicale et en particulier la dissection postérieure. Le promontoire sera dégagé des anses grêles qui le recouvrent. Deux pinces non traumatiques tractent les anses qui seront positionnées dans la cavité abdominale. La fixation utérine (fig. 3) se fait par l'intermédiaire d'un fil avec une aiguille droite qui passe à travers la paroi abdominale antérieure (en sus-pubien) puis à travers le corps utérin. Elle est pour nous systématique et permet d'ouvrir la cavité pelvienne. La fixation du côlon sigmoïde (optionnelle) permet de compléter cette exposition (fig. 4). Une aiguille droite est passée à travers la paroi abdominale antérieure, en fosse iliaque gauche en dessous et en dehors du trocart de l'aide. Il est conseillé de fixer le sigmoïde par l'intermédiaire de plusieurs franges afin d'éviter une déchirure. Certains chirurgiens préfèrent utiliser des fils de fixation comportant une extrémité en T (ancre en plastique) 
limitant les manipulations intracorporelles avec l'aiguille droite mais dont le coût est à mettre en balance avec le bénéfice apporté.

\section{Abord du promontoire}

On démarre habituellement l'intervention par l'incision du péritoine pariétal postérieur en regard du promontoire (fig. 5). Cet espace se situe dans un triangle situé en dessous de la bifurcation des artères iliaques primitives. L'exposition du promontoire est facile lorsqu'il est saillant. Dans le cas contraire, il ne faut pas hésiter à remonter l'incision du péritoine jusqu'à la bifurcation iliaque pour repérer les éléments vasculaires. Il faudra en particulier bien repérer la veine iliaque primitive gauche qui a une orientation beaucoup plus verticale que la droite et qui sera donc plus proche du point de fixation sur le ligament prévertébral. La veine iliaque peut être aplatie contre le promontoire en raison de la position de Trendelenburg et du pneumopéritoine. Il est donc dangereux de passer une aiguillée, ou pire une agrafe dans le ligament prévertébral sur le promontoire sans avoir visualisé la totalité de la zone de fixation. En cas de doute, il ne faut pas hésiter à diminuer la pression du pneumopéritoine, ce qui permettra de mieux visualiser le relief de la veine iliaque. Afin d'éviter un saignement lié à une blessure des vaisseaux présacrés qui cheminent sur le promontoire, il peut être nécessaire de les coaguler préventivement à l'aide d'une pince bipolaire. On peut enfin recommander d'éviter une dissection aveugle dans la concavité du sacrum ou un saignement veineux serait difficile à contrôler.

\section{Dissection du péritoine du promontoire jusqu'au cul-de-sac de douglas}

Avant de démarrer cette dissection, il est prudent de repérer l'uretère droit dans son trajet pelvien car il existe potentiellement un risque de traumatisme de ce dernier à ce niveau. L'uretère est souvent visualisé au niveau de son croisement avec l'artère iliaque droite avant de plonger dans la concavité pelvienne. Certaines variations anatomiques ou certains antécédents chirurgicaux pelviens comme une hystérectomie peuvent avoir rendu le trajet de l'uretère plus médian et donc plus proche de la zone de dissection. En cas de doute, pour faciliter son repérage, on pourra stimuler la diurèse par une injection de diurétique permettant d'augmenter le péristaltisme urétéral.

Plusieurs astuces peuvent faciliter la dissection du péritoine et ultérieurement celui de sa fermeture. Il faudra tout d'abord s'assurer d'être dans un plan sous 
péritonéal très superficiel (fig. 6) pour pouvoir faire un décollement par traction sans faire saigner le réseau vasculaire sous péritonéal et ensuite cheminer entre méso rectum à gauche et paroi pelvienne à droite (fig. 7). Il est possible d'introduire 2 pinces non traumatiques dans l'espace de dissection et les écarter progressivement jusqu'au niveau du ligament utéro sacré droit dans sa portion juxta utérine (fig. 8). Cette dissection est facilitée par la suspension préalable de l'utérus à la paroi abdominale antérieure. Le péritoine peut ensuite être incisé sur toute sa longueur depuis le promontoire jusqu'au ligament utéro sacré droit mais aussi être conservé intact pour passer les prothèses sous le péritoine « en pont » jusqu'à son ouverture en avant du promontoire.

Pour poursuivre la dissection au niveau de la cloison recto vaginale, l'exposition sera aidée par une valve malléable à bord mousses (idéalement de largeur $5 \mathrm{~cm}$ ) introduite dans le cul-de-sac vaginal postérieur. Cette valve sera incurvée à $90^{\circ}$ de manière à exposer la paroi vaginale postérieure (verticalisation). Une traction postérieure sur le péritoine du cul-de-sac de douglas permettra d'ouvrir l'espace recto vaginal (fig. 9). L'incision péritonéale sera poursuivie le long du bord interne des ligaments utéro sacrés et rejoindra du côté droit l'incision du péritoine préalablement réalisée à partir du promontoire.

\section{Dissection de la cloison recto vaginale}

On réalise une incision arciforme du péritoine du cul-de-sac de douglas (fig. 10) poursuivie par une dissection médiane entre rectum et paroi vaginale (fig. 11) avant d'amorcer la dissection latéralement vers les muscles releveurs (fig. 12). Ne pas disséquer les faces latérales et la face postérieure du rectum permet d'éviter une dénervation [5] et de limiter le risque d'induire des troubles de l'exonération.

L'exposition de cet espace sera facilitée par une traction divergente entre rectum vers l'arrière (pince de l'aide prenant la berge du péritoine du douglas) et vagin vers le haut (valve vaginale). Une pince à bord mousse prenant contact avec la paroi vaginale (servant de guide) permettra d'ouvrir progressivement l'espace recto vaginal jusqu'au niveau du canal anal (visualisé sous forme d'un aspect blanc nacré). Le trajet du rectum à partir de la zone d'ouverture péritonéale présente une première portion horizontale (voire ascendante) avant de plonger vers le canal anal. Cette première partie de dissection est donc à risque de plaie rectale si le chirurgien ne prend pas garde à suivre la paroi vaginale. 
Ce n'est donc qu'après avoir exposé la totalité de l'espace recto vaginal que le chirurgien pourra sans aucune difficulté mettre en évidence latéralement la partie distale des muscles releveurs. Le plancher périnéal a dans cette partie une orientation ascendante. L'ouverture de l'espace pararectal se fera au mieux à l'aide d'une pince forceps introduite par le trocart du côté opposé au muscle releveur à exposer : ouverture des mords très progressivement puis traction divergente entre 2 pinces pour avoir un espace suffisant pour la fixation. Cette stratégie de dissection permet de respecter au mieux les pédicules vasculo-nerveux dans le méso rectum latéral

\section{Fixation de la Prothèse postérieure :}

Les deux matériaux recommandés pour la promontofixation par cœlioscopie sont représentés par les prothèses de polyester et de polypropylène. Nous conseillons l'utilisation d'une prothèse non résorbable macroporeuse et de faible grammage (30 à $40 \mathrm{~g} / \mathrm{m}^{2}$ ). Ces prothèses dites « light » offrent une résistance équivalente aux prothèses de plus fort grammage, à condition de ne pas descendre au-dessous d'une limite d'environ $30 \mathrm{~g} / \mathrm{m}^{2}$ avec l'avantage d'une bien meilleure souplesse. Cette propriété, valable aussi bien avec le polypropylène qu'avec le polyester leur permet d'épouser parfaitement l'espace de dissection. Le large maillage permet de visualiser le tissu lors du passage de l'aiguille à travers la prothèse donnant une sécurité quand à la profondeur de la fixation.

Un autre conseil est de fixer la prothèse dans la partie la plus basse du muscle releveur $[6,7,8]$. Ce point est un des plus difficiles de l'intervention et certaines règles permettent de le faciliter (fig. 13A). L'aide récline le rectum vers l'intérieur et l'utilisation d'un 2eme porte-aiguille permettra à l'opérateur d'attraper plus facilement la pointe de l'aiguille à sa sortie de son passage musculaire. Le point de fixation à ce niveau ne devra pas être trop serré en raison du risque de douleurs périnéales induites

(fig. 13B). La prothèse prédécoupée comporte une légère échancrure médiane permettant de passer en pont sur le rectum sans striction. Cette échancrure ne doit cependant pas être trop prononcée si l'on veut couvrir la partie basse médiane du rectum. Dans tous les cas, un point prenant la partie médiane de la prothèse à la partie la plus déclive de la dissection permet d'éviter une récidive de rectocèle basse sous la prothèse (fig. 14). La prothèse sera ensuite tractée vers l'utérus et ainsi plaquée contre la paroi vaginale et fixée en haut et latéralement à la partie juxta utérine des ligaments 
utéro sacrés. Un point médian plus profond prenant l'isthme utérin postérieur permet d'assurer la solidité de la fixation (fig. 15).

\section{Dissection de l'espace vésico vaginal}

L'exposition est assurée par une traction divergente entre les plans à dissocier : traction du péritoine pré vésical vers la paroi abdominale antérieure et traction de l'utérus en direction du promontoire. Il se crée ainsi une bride péritonéale médiane partant de l'isthme utérin antérieur que l'on pourra facilement inciser à 1 ou $2 \mathrm{~cm}$ audessus de l'isthme utérin (fig. 16). L'incision péritonéale sera prolongée latéralement jusqu'au niveau des ligaments larges, dépassant de chaque côté l'isthme utérin. L'incision sera un peu plus longue du côté droit pour permettre le passage de la prothèse (fenêtre dans le ligament large). La valve vaginale (lame malléable) est alors positionnée dans le cul-de-sac vaginal antérieur et poussée vers la tête de la patiente. Il est souhaitable que les bords de la valve soient légèrement arrondis pour ne pas risquer de blesser la paroi vaginale et que la largeur de la valve corresponde à la largeur de dissection soit $5 \mathrm{~cm}$ (correspondant à la largeur de la prothèse prédécoupée). La traction vésicale vers la paroi abdominale à l'aide d'une pince forceps permet de tendre les adhérences entre vessie et vagin qui seront coagulées et coupées (fig. 17). La dissection sera poursuivie jusqu'à apercevoir le relief du ballon de la sonde vésicale. Une dissection trop hémorragique signifie le plus souvent une dissection trop proche de la paroi vésicale. Il faudra dans ce cas revenir à la partie haute de la dissection vaginale et prendre contact avec le vagin (zone blanc nacrée) et reprendre la dissection a son contact avec les ciseaux ou la pince forceps.

\section{Fixation de la prothèse antérieure}

Le choix de la prothèse est essentiel et nous conseillons d'utiliser, comme pour l'étage postérieur, une prothèse en polypropylène ou polyester à faible grammage « light » sans descendre en dessous de $30 \mathrm{~g} / \mathrm{m} 2$. Le polypropylène présente l'avantage d'une meilleure intégration tissulaire limitant le risque infectieux en cas d'exposition prothétique. Le large maillage de cette prothèse lui permet d'une part de bien épouser la surface de la paroi vaginale et d'autre part, de visualiser la profondeur des points lors de la fixation de cette dernière : points superficiels sur le vagin pour étaler la prothèse (fig. 18) et points profonds sur l'isthme antérieur (fig. 19) Il y a un consensus sur le 
positionnement et la fixation de la prothèse antérieure dans les différentes publications. Il convient de fixer la prothèse à la partie haute sur l'isthme utérin afin d'éviter l'effet «piston » avec un prolapsus utérin isolé secondaire $[9,10]$. Concernant la fixation sur la face antérieure du vagin, aucune étude comparative ne permet de dire quelle doit être l'étendue de la dissection vaginale sous vésicale. La plupart des grandes séries rétrospectives conseillent une dissection assez médiane partant de l'isthme utérin jusqu'au relief du ballon de la sonde vésicale et ce sur une largeur d'environ 4 à $5 \mathrm{~cm}$. La plupart des grandes séries rétrospectives décrivent une fixation au vagin à l'aide de fils résorbable ou non et conseillent de ne pas traverser la paroi du vagin mais aucune étude n'a évalué la profondeur du système de fixation (gant, valve, toucher, examen sous spéculum...) $[6,7,8]$.

\section{Passage de la prothèse à travers le ligament large droit :}

Le passage unilatéral droit est beaucoup plus aisé que le passage bilatéral sans modifier l'efficacité de la technique de promontofixation. Ce temps relativement simple peut exposer à certaines complications que quelques astuces techniques permettent d'éviter. Tout d'abord, lors de l'ouverture du péritoine inter vésico utérin (pour la dissection de la cloison vésico vaginale) il faudra poursuivre l'incision du péritoine latéralement du côté droit, en longeant le ligament rond. Nous avons ainsi ouvert le feuillet antérieur du ligament large droit. Il faut alors demander à l'aide opératoire de tracter le ligament rond vers le haut, ce qui permet une dissection aisée entre les 2 feuillets du ligament large. On a alors une excellente vision des différents éléments à éviter : artère utérine en dedans, uretère en bas et vaisseaux ovariens en dehors

(fig. 20). Lors de la remise en traction sur l'utérus, on visualise par transparence l'espace de dissection et on peut aisément ouvrir le feuillet postérieur au-dessus de la traversée urétérale sans risque vasculaire. La prothèse antérieure grâce à ce passage sécurisé arrive à proximité du ligament utéro sacré droit. Le trajet de dissection péritonéal postérieur peut ainsi être rejoint pour que les 2 prothèses puissent être réunies pour une péritonisation commune jusqu'au promontoire. (Fig. 21)

\section{Péritonisation postérieure :}

La fermeture du péritoine postérieure peut être difficile si l'espace de décollement entre méso-rectum et paroi pelvienne est insuffisant. Ceci survient en cas 
de dissection trop latérale sur la paroi pelvienne ou au contraire trop médiane vers le méso-rectum sans dissociation de l'espace sous péritonéal. Pour faciliter cette fermeture, il faudra partiellement libérer la traction sur l'utérus, ce qui permet de rapprocher les berges au niveau du douglas. L'utilisation d'un fil cranté (V lock) facilite la fermeture péritonéale. Il est préférable de réaliser la fermeture du péritoine postérieur dans sa partie pelvienne (fig. 22) avant la fixation des prothèses au promontoire. En effet, la fixation des prothèses nécessite une traction sur ces dernières, pouvant entraîner une difficulté pour l'affrontement des berges pour la fermeture du péritoine postérieur. Enfin, la fermeture péritonéale peut modifier la tension exercée sur les prothèses si celles-ci sont déjà fixées au promontoire. Celles-ci pourront être alors trop tendues.

\section{Fixation des prothèses au promontoire}

Il est important de préparer le site de fixation des prothèses sur le promontoire. Il faut pour cela, prendre contact et visualiser le ligament recouvrant le promontoire. Il faut toujours se méfier d'une veine iliaque G pré sacré et aplatie sur le promontoire par le pneumopéritoine (bifurcation veineuse basse) ou à une veine sacrée médiane qui en cas de traumatisme peut se rétracter sous le promontoire (hématome). Il nous paraît utile de réaliser la fixation avec des aiguilles à faible courbure pour prendre le ligament de manière large mais pas trop profonde (fig. 23) et d'utiliser des fils non résorbables $[7,8]$. Certains chirurgiens préfèrent utiliser une fixation au promontoire à l'aide de Tackers car aucune étude prospective et comparative n'a démontré son infériorité. Il existe cependant des études anatomiques ayant démontré que cette fixation offre une moindre résistance à la traction que la fixation à l'aide de fils [11]. D'autre part, les risques potentiels de cette technique en cas de lésion vasculaire en particulier veineuse et de la difficulté d'ablation de ce matériel en cas d'infection prothétique nous font clairement préférés la fixation à l'aide de fils.

La fixation des prothèses doit se faire sans tension excessive. Elles doivent être appliquées sur le ligament pré vertébral et maintenus par l'aide opératoire pendant leur fixation par le chirurgien. Une tension insuffisante peut être au besoin ajustée par le deuxième point de fixation. En fin de procédure, la prothèse doit pouvoir être mobilisée de 1 à $2 \mathrm{~cm}$. Une prothèse trop tendue risque d'entraîner des douleurs pelviennes et met la fixation à l'épreuve avec un risque accru de déchirure et donc de récidive. 
On termine ensuite la fermeture du péritoine pariétal postérieur qui vient recouvrir la fixation des prothèses sur le promontoire (fig. 24)

\section{Péritonisation antérieure}

L'intervention se termine par la fermeture du péritoine inter vésico-utérin. Ce temps est beaucoup plus aisé que celui du péritoine postérieur mais ne doit pas être négligé si on ne veut pas exposer la patiente à un risque d'occlusion par incarcération d'une anse grêle et surtout du risque d'érosion de la paroi digestive au contact d'une prothèse non résorbable. Il faut notamment couper le fil à la fin du surjet au ras du péritoine.

L'intervention se termine par le repositionnement des anses grêles dans la cavité pelvienne, une fois la patiente remise à plat puis au retrait des différents trocarts sous contrôle de la vue pour ne pas méconnaître une plaie vasculaire pariétale qui serait responsable d'un saignement postopératoire pouvant être conséquent et nécessiter une reprise chirurgicale.

\section{CONCLUSION}

La promontofixation est une intervention réalisée par laparotomie depuis de très nombreuses années. La technique chirurgicale a démontré son efficacité dans le traitement du prolapsus, et ce, sur un grand nombre de patientes et avec un recul important. L'utilisation de prothèses non résorbables permet d'obtenir de très bons résultats, durables et comportant peu de complications y compris en termes d'exposition et d'infection de prothèses. La technique laparoscopique reproduit les mêmes temps opératoires que ceux réalisées par laparotomie avec une moindre morbidité pariétale. Elle offre une meilleure vision grâce au grossissement optique et un net avantage pour la dissection des espaces sous-péritonéaux grâce au pneumopéritoine. Cela facilite grandement la mise en place des prothèses, en particulier postérieure. De nombreuses variantes techniques se sont développées et rendent parfois difficile l'analyse des résultats. Une mise au point sur cette technique en vue d'une standardisation s'avère donc indispensable. 
Liens d'intérêt :

LW : liens d'intérêts avec les laboratoires Porges Coloplast, Boston Scientific et AB Medica ;

AV : Contrat d'expert scientifique, contrat dans le cadre d'une recherche, contrat de consultant : ASTELLAS Pharma. Contrat d'expert scientifique, contrat dans le cadre d'une recherche, contrat de consultant : Boston Scientific SAS

CT : aucun lien d'intérêt

Points essentiels à retenir :

- Dissection de la cloison recto vaginale :

- Ne disséquer en pararectal que lorsque la dissection atteint le bas rectum (blanc nacré)

- La dissection se fera à $2 \mathrm{~h}$ et $10 \mathrm{~h}$ (partie ascendante des muscles releveurs).

- Cette dissection latérale très basse permet d'éviter la vascularisation rectale

- Fixation de la Prothèse postérieure :

- Ne pas serrer trop fort le point sur les muscles releveurs (douleur périnéale induite)

- Mettre un point médian fixé à la partie basse de la prothèse (récidive de rectocèle par glissement du rectum sous la prothèse)

- Fixer la prothèse à la partie haute sur les ligaments US (étalement de la prothèse) et solidement sur l'isthme utérin (médian)

- Fixation des prothèses au promontoire :

- Le bord droit du promontoire est plus libre que le gauche (Méso rectum).

- Le ligament est plus épais sur la partie haute horizontale du promontoire

- La péritonisation du douglas préalable à la fixation au promontoire facilite le réglage de la tension des prothèses

- Ne pas oublier de relâcher la traction sur l'utérus lors de la fixation sur le promontoire

- Régler soigneusement la tension sur les prothèses lors de la fixation au promontoire. 


\section{Références}

1. Ganatra AM, Rozet F, Sanchez-Salas R, et al. The current status of laparoscopic sacrocolpopexy: a review. Eur Urol 2009; 55 (5): 1089-103.

2. Gadonneix P, Ercoli A, Salet-Lizée D, et al. Laparoscopic sacrocolpopexy with two separate meshes along the anterior and posterior vaginal walls for multi compartment pelvic organ prolapse. J Am Assoc Gynecol Laparosc 2004; 11: 29— 35.

3. Rozet F , Mandron E, Arroyo C, et al. Laparoscopic sacral colpopexy approach for genito-urinary prolapse: experience with 363 cases. Eur Urol 2005; 47:230—6.

4. Mandron E, Bryckaert PE. Prolapsus et colpocèle antérieure. Double promontofixation cœlioscopique. Technique. Ann Urol (Paris) 2005 ; 39 (6) : $247-56$.

5. D’Hoore A, Penninckx F. Laparoscopic ventral rectopexy for rectal prolapse: surgical technique and outcome for 109 patients. Surg Endosc (2006) 20: 19191923Higgs PJ, Chua HL, Smith AR. Long-term review of laparoscopic sacrocolpopexy. Br J Obstet Gynaecol 2005 ; 112 : 1134-8.

6. Higgs PJ, Chua HL, Smith AR. Long-term review of laparoscopic sacrocolpopexy. Br J Obstet Gynaecol 2005; 112 : 1134-8

7. Ross JW, Preston M. Laparoscopic sacrocolpopexy for severe vaginal vault prolapse: five-year outcome. J Minim Invasive Gynecol 2005; 12 : 221—6.

8. Sarlos D, Brandner S, Kots L, Gygax N, Schaer G. Laparoscopic sacrocolpopexy for uterine and post-hysterectomy prolapse: anatomical results, quality of life and perioperative outcome: a prospective study with 101 cases. Int Urogynecol J Pelvic Floor Dysfunct 2008; 19: 1415-22.

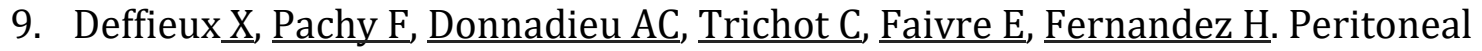
closure using absorbable knotless device during laparoscopic sacrocolpopexy. I Gynecol Obstet Biol Reprod (Paris). 2011 Feb;40(1):65-7.

10. Quiroz LH, Gutman RE, Shippey S, et al. Abdominal sacrocolpopexy: anatomic outcomes and complications with Pelvicol and autologous and synthetic graft material. Am J Obstet Gynecol 2008:198-557

11. Boukerrou M, Orazi G, Nayama M, Boodhun R, Crépin G, Cosson M. Promontofixation procedure: use of non-absorbable sutures or Tackers? J Gynecol Obstet Biol Reprod (Paris) 2003; 32 (6) : 524-8. 
Légendes des figures

Fig 1 : Installation de la patiente

Fig 2 : Open coelioscopie

Fig 3 : Fixation utérine

Fig 4 : Fixation sigmoidienne

Fig $5 a$ et $5 b:$ Abord du promontoire

Fig 6 : Dissection du péritoine pariétale postérieur

Fig 7 : Décollement du péritoine pariétal postérieur

Fig 8 : Décollement péritonéal jusqu'au ligament utéro sacré

Fig 9 : Incision du péritoine du cul-de-sac de Douglas

Fig 10 : Incision arciforme du cul-de-sac de Douglas

Fig 11 : décollement recto vaginal

Fig 12 : libération latéro rectale (muscles releveurs)

Fig 13a,13b, 13c : Fixation latérale basse de la prothèse aux muscles releveurs

Fig 14a et 14b : Fixation médiane de la prothèse à la jonction recto-vaginale

Fig 15a et 15b : Fixation médiane de la prothèse à l'isthme utérin postérieur

Fig 16 : Incision du péritoine pariétal antérieur

Fig $17 \mathrm{a}$ et $17 \mathrm{~b}$ : Dissection du plan vésico vaginal antérieur

Fig 18a et 18b : Fixation de la prothèse antérieure à la paroi vaginale

Fig 19a et 19b : Fixation de la prothèse antérieure à l'isthme utérin antérieur

Fig 20 : Libération du feuillet antérieur du ligament large droit

Fig 21 : Incision puis traversée du ligament large droit par la prothèse antérieure

Fig 22a, 22b, 22c : Début de péritonisation postérieure

Fig 23a et 23b: Fixation des prothèses sur le promontoire

Fig 24 : fin de péritonisation postérieure 
Fig. 1a: Installation de la patiente

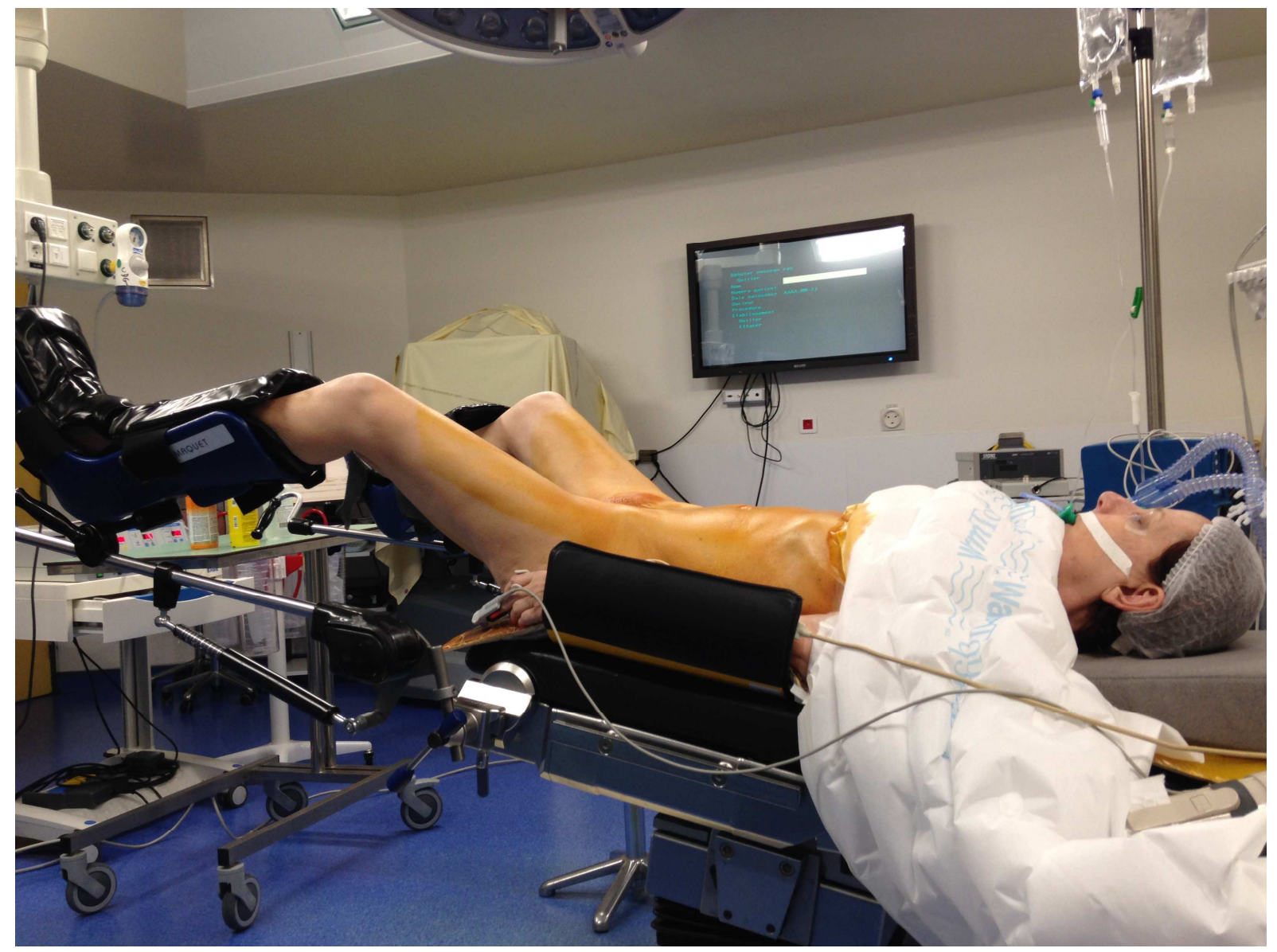


Fig. 1b Open coelioscopie

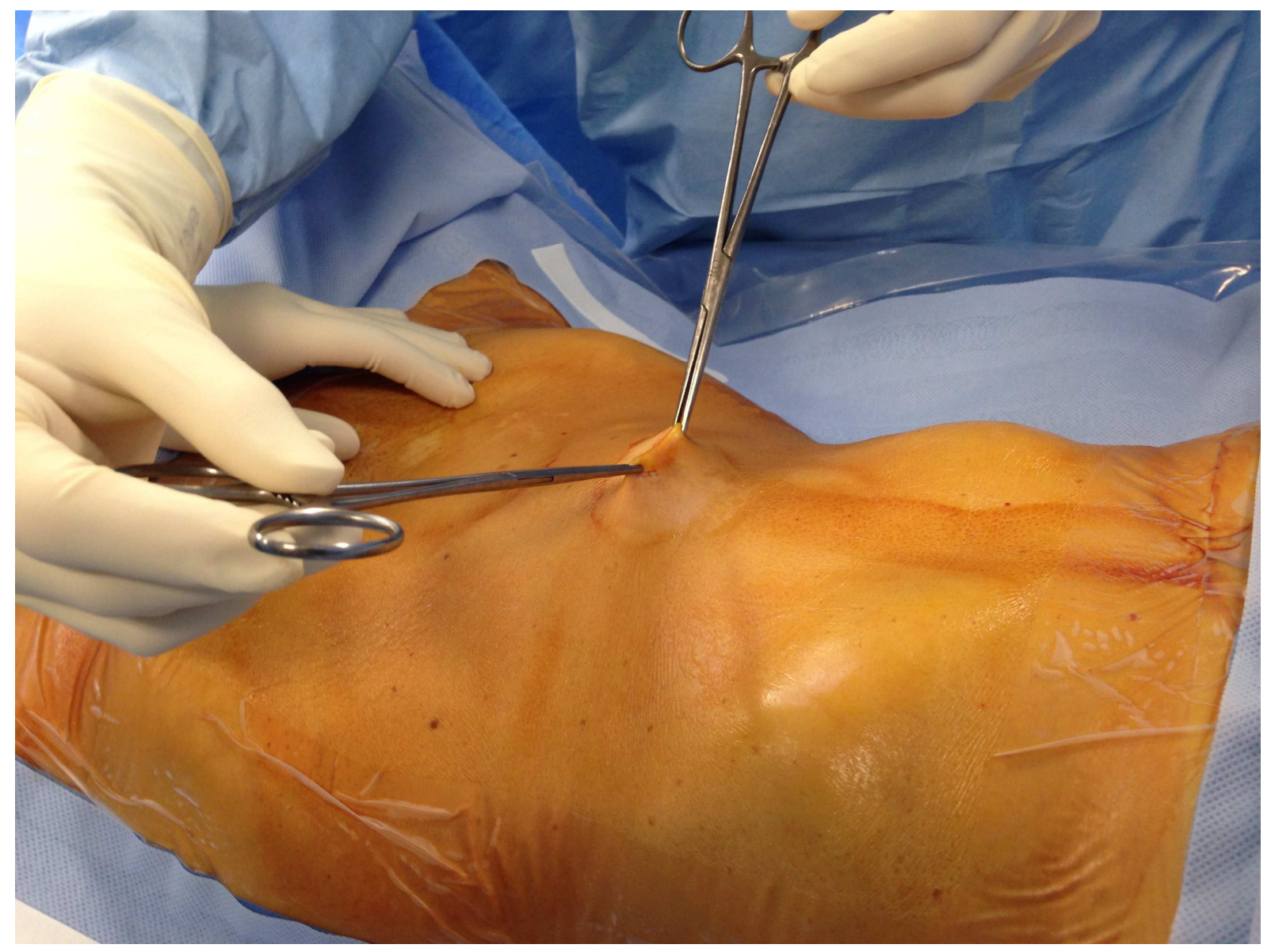




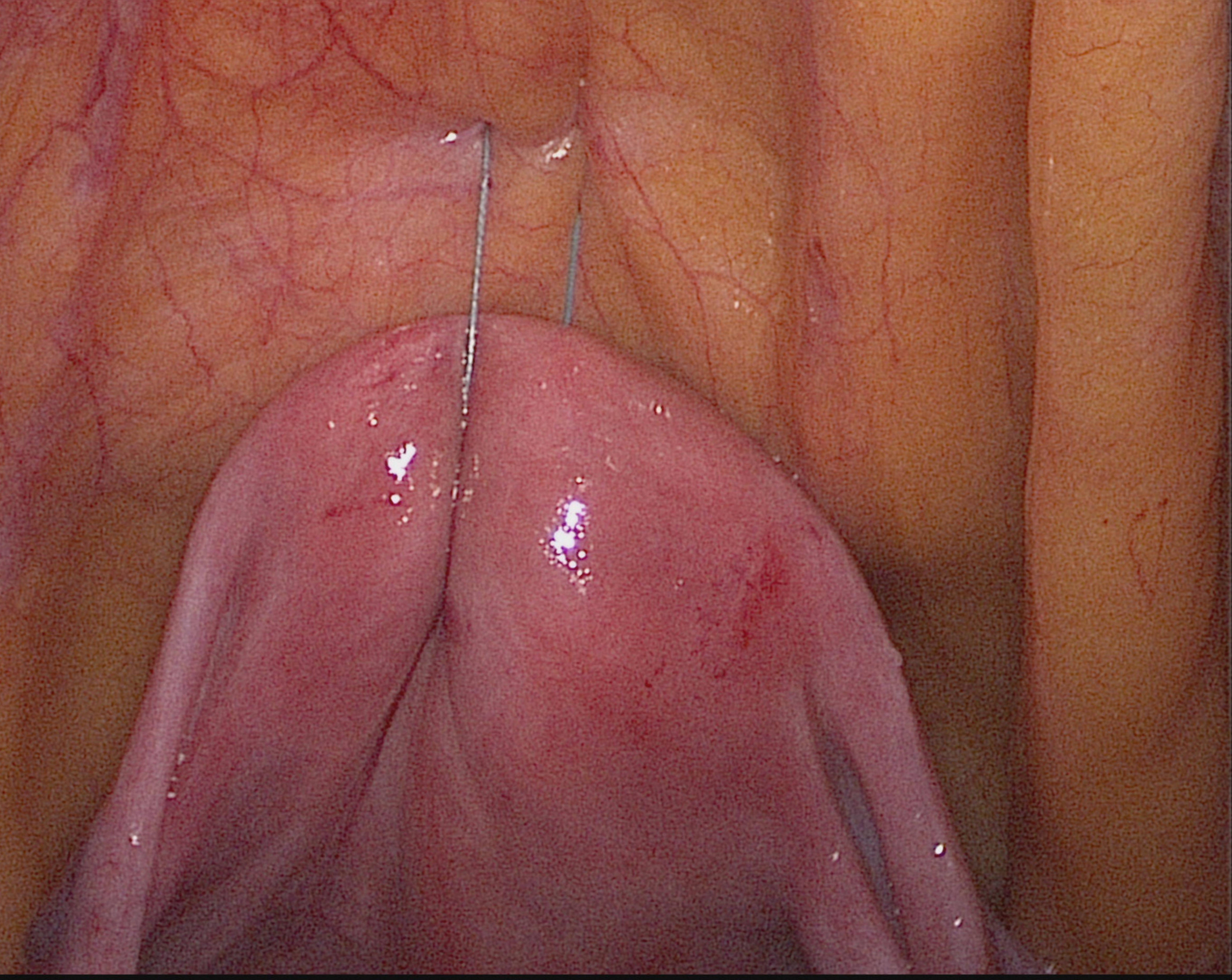




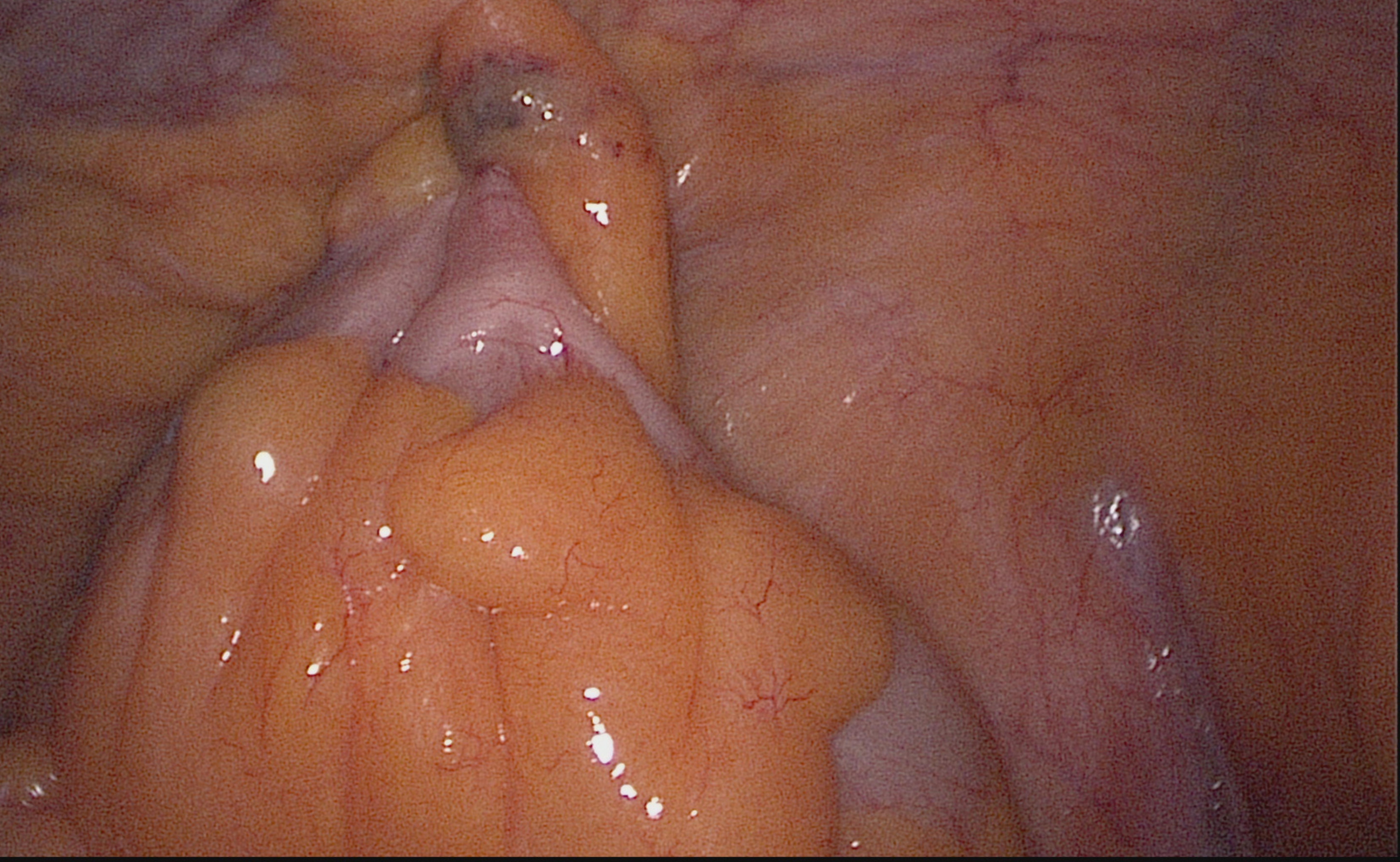




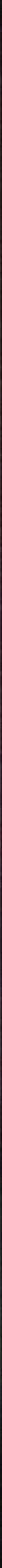




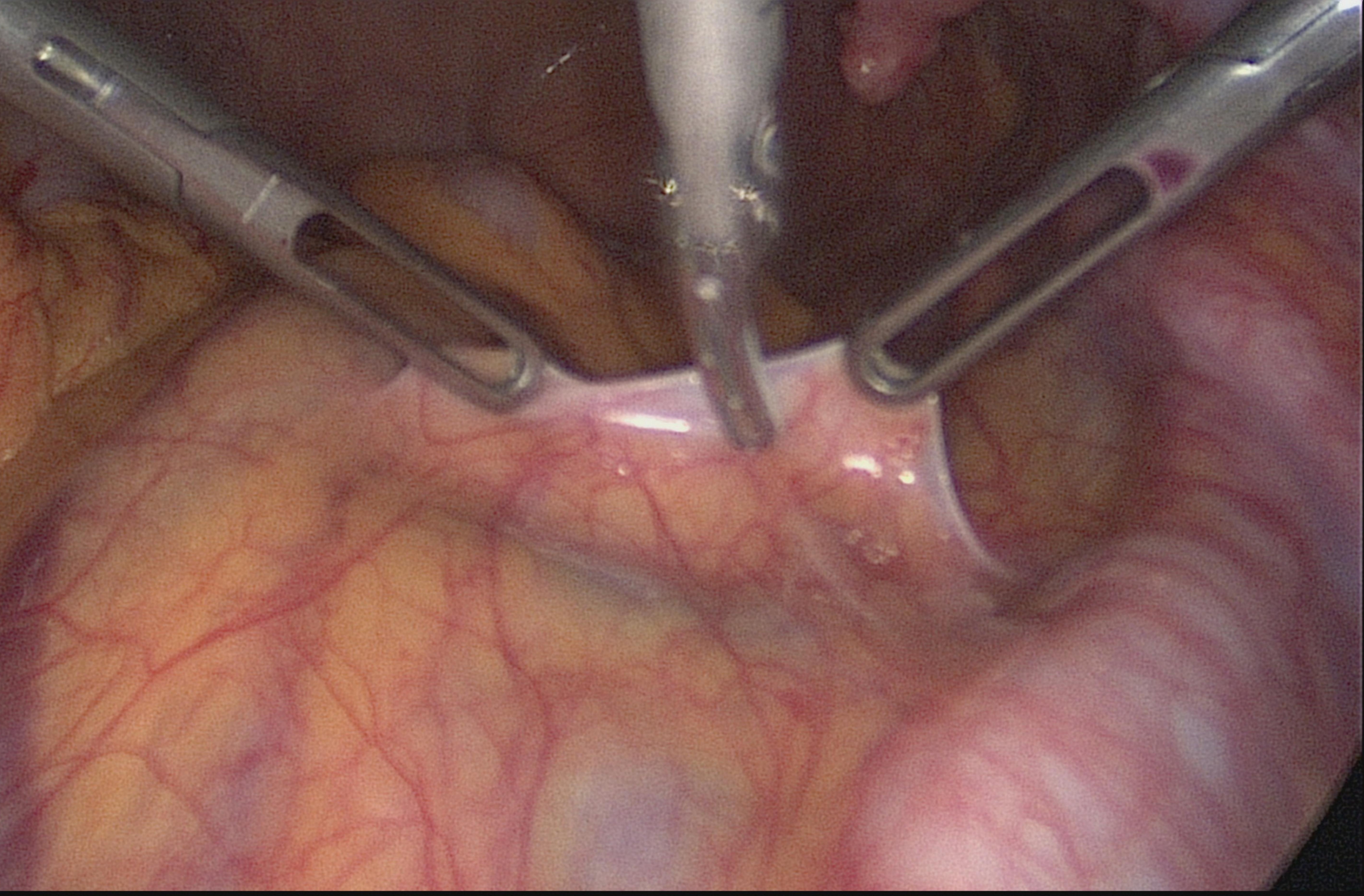





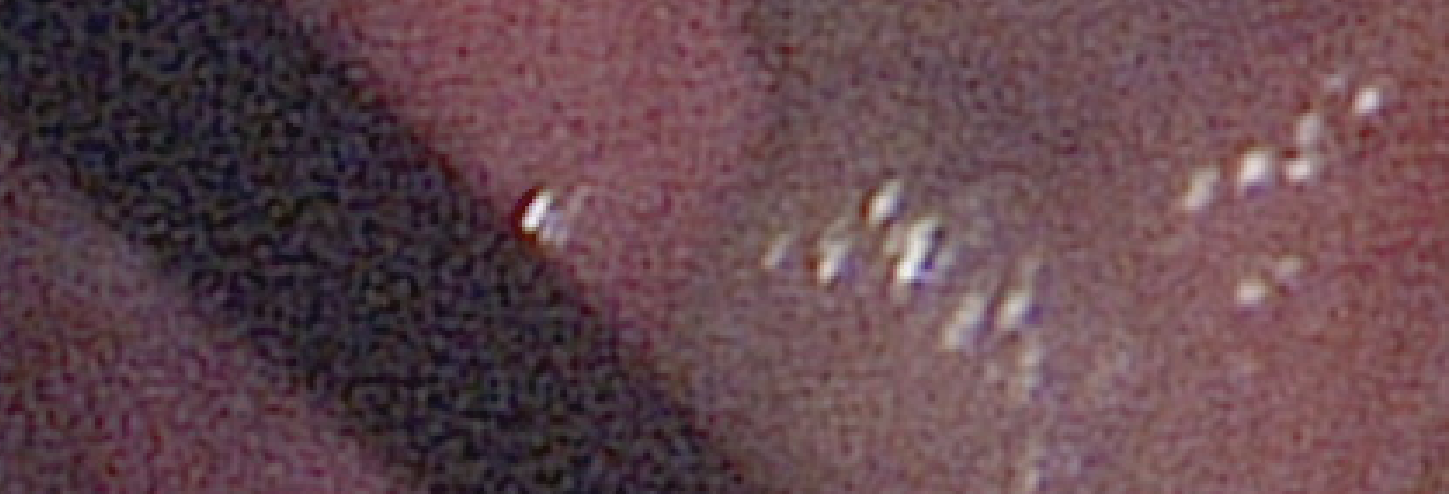

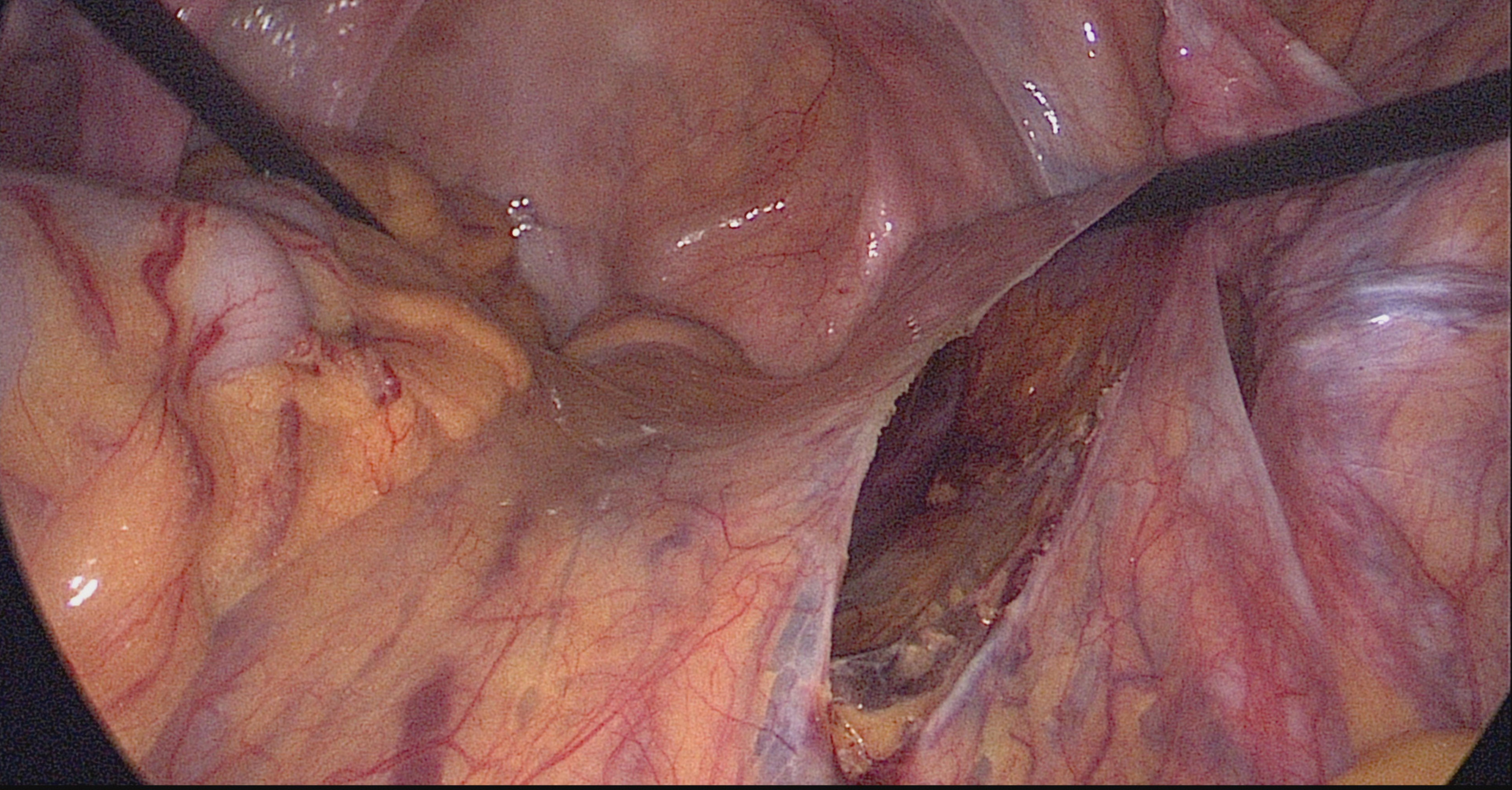




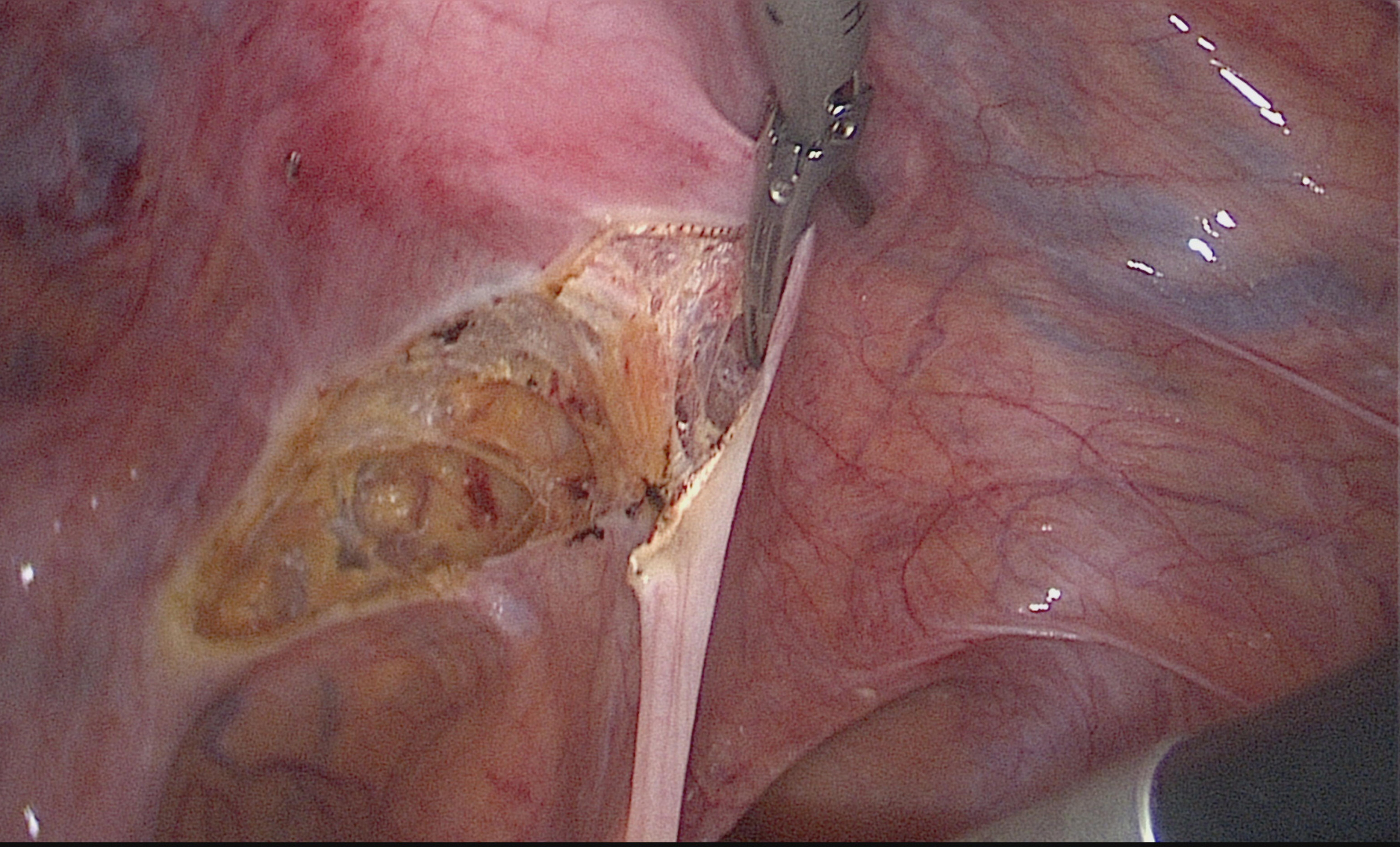




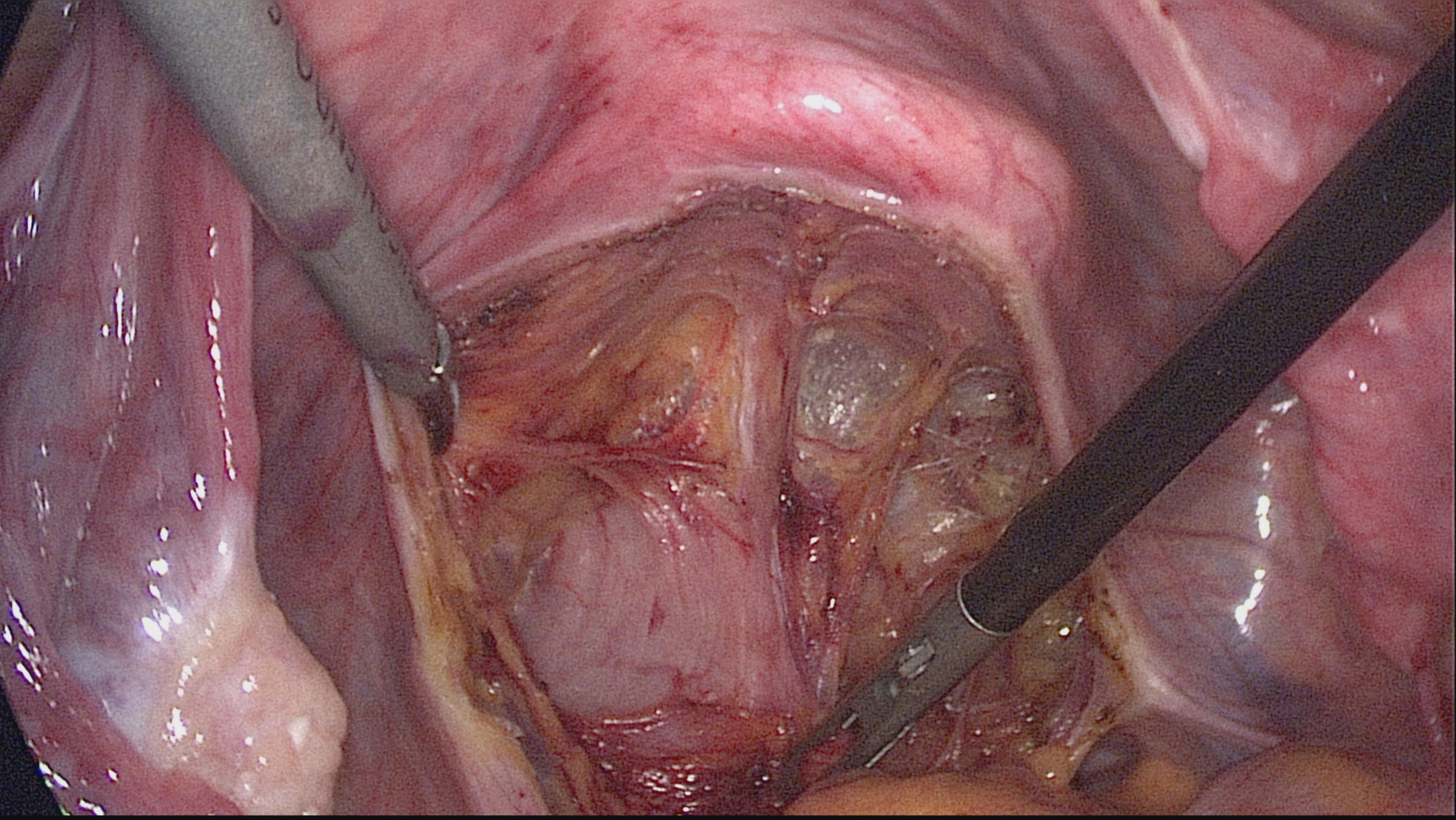




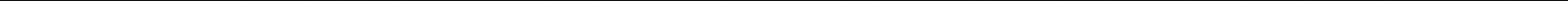




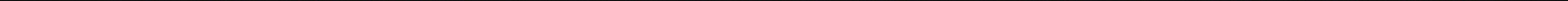




\section{$\Leftrightarrow$}

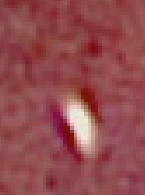

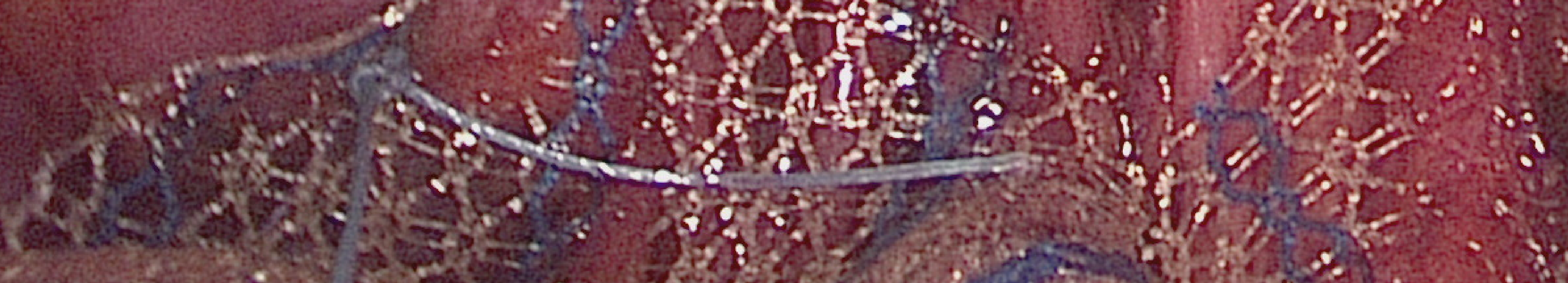

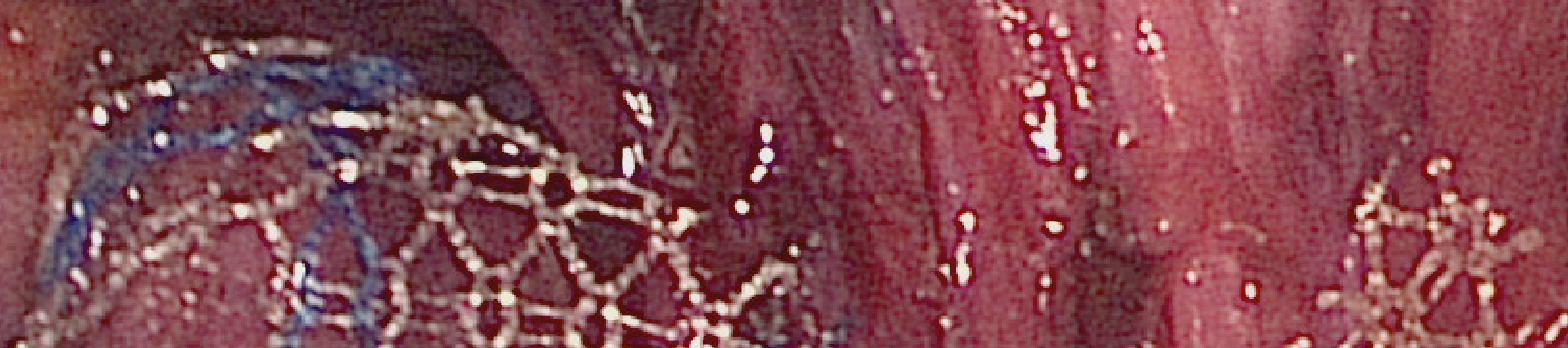

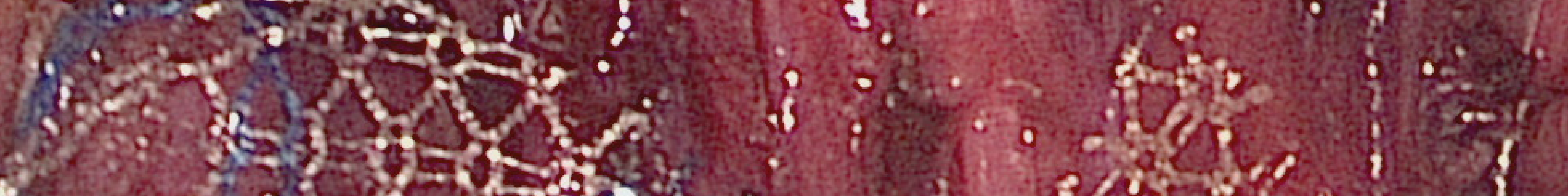

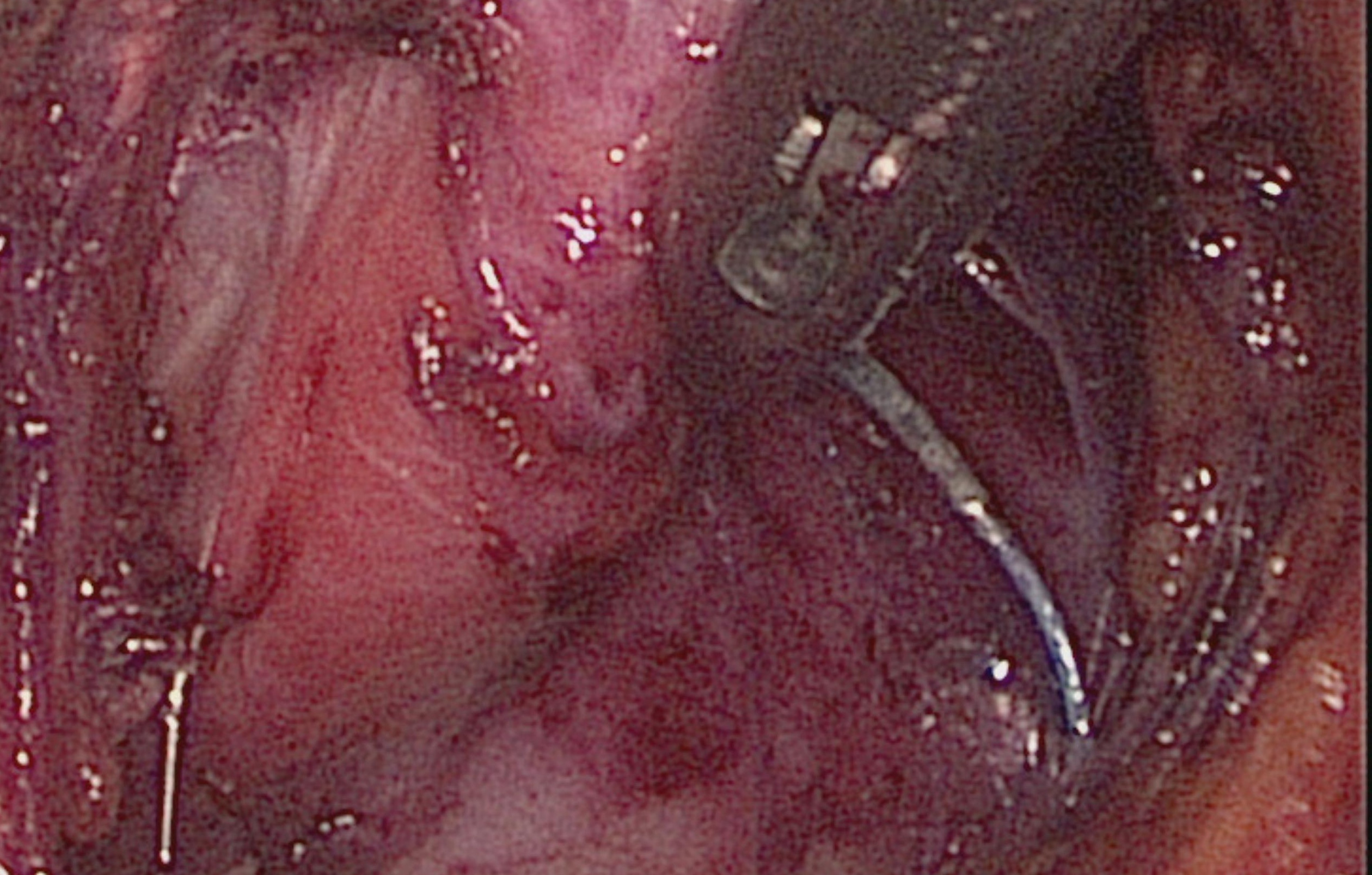

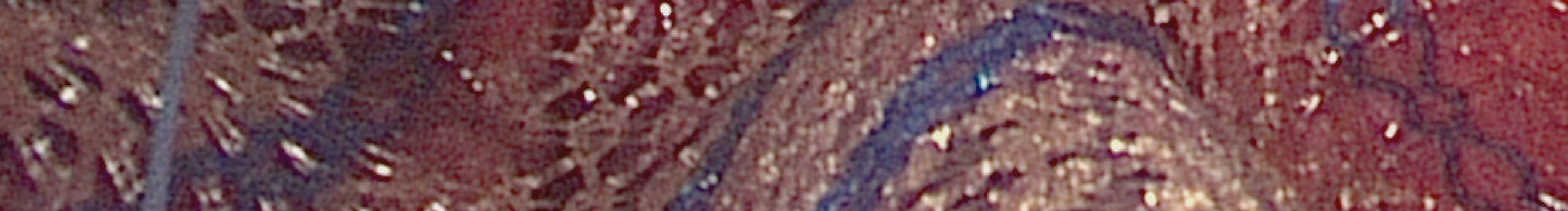
$-4$ 
S.:.

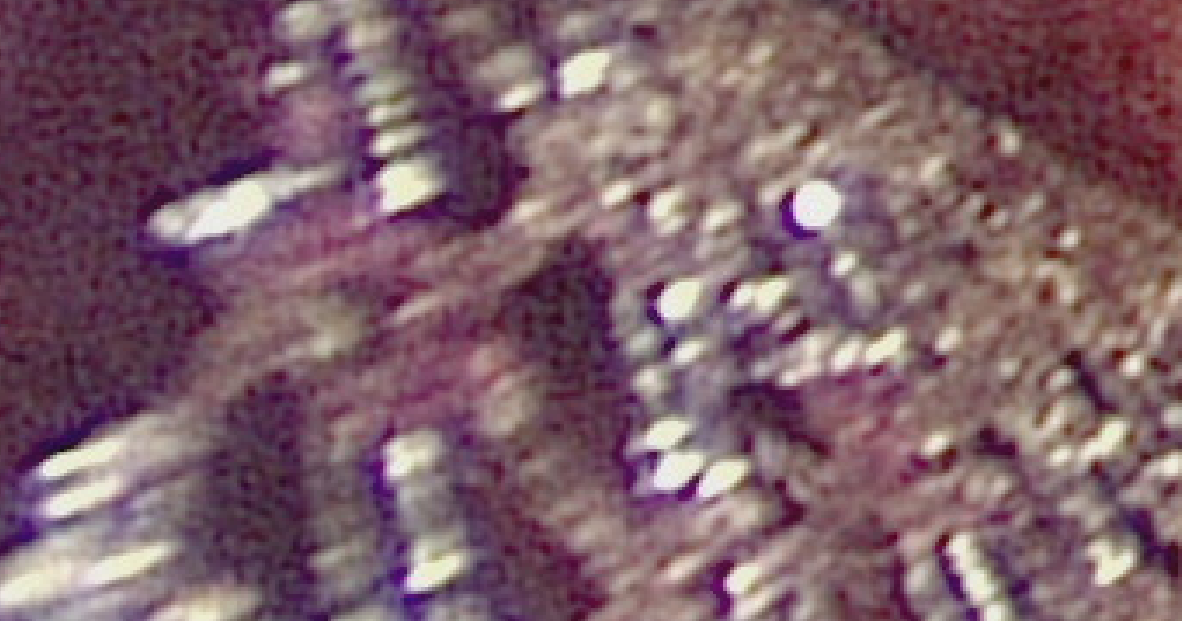

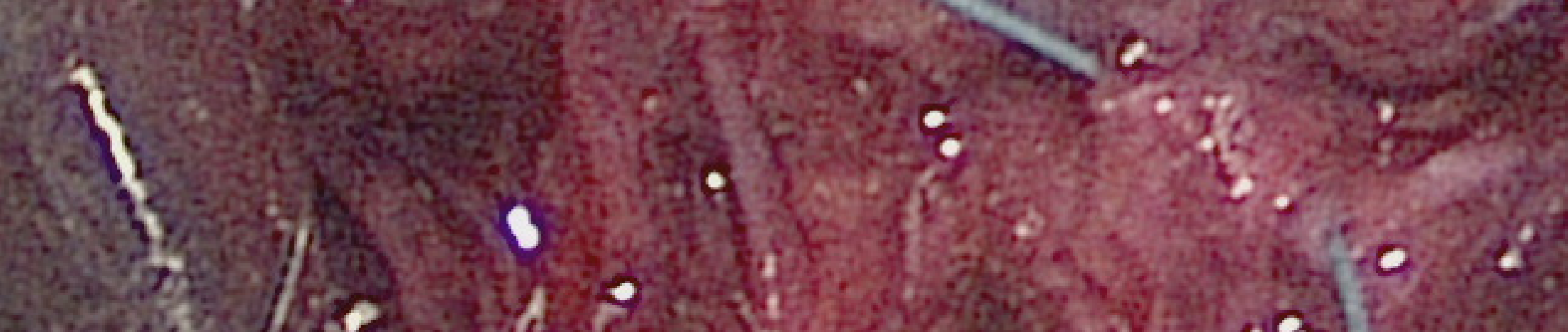

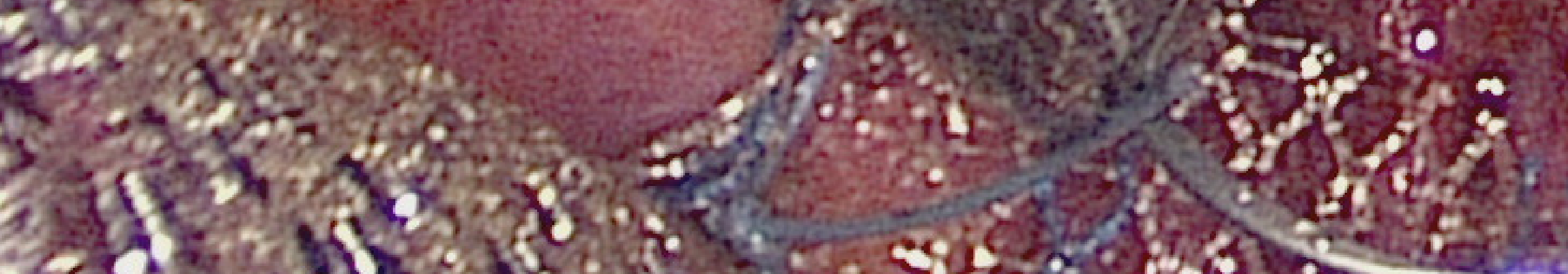

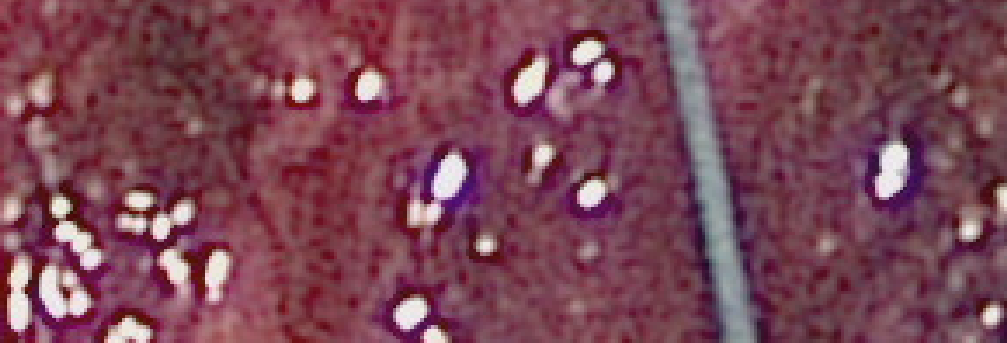
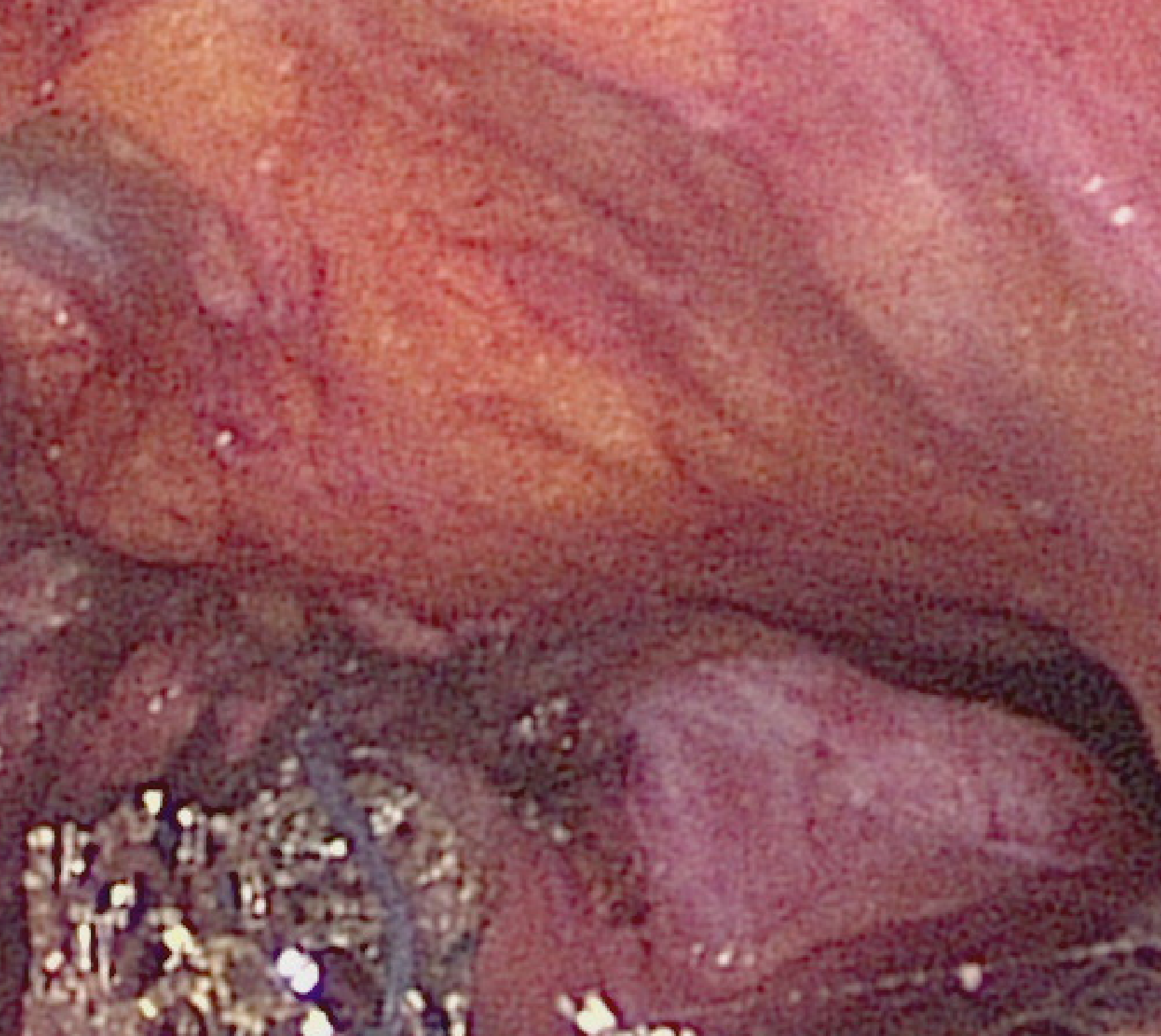
(c)

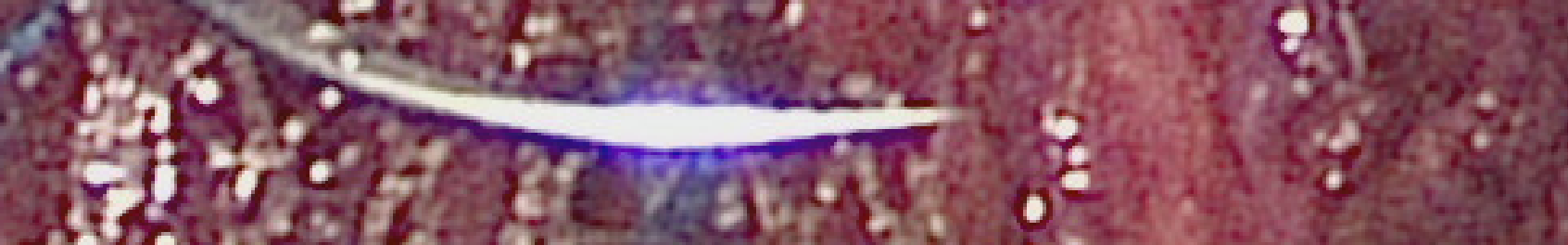

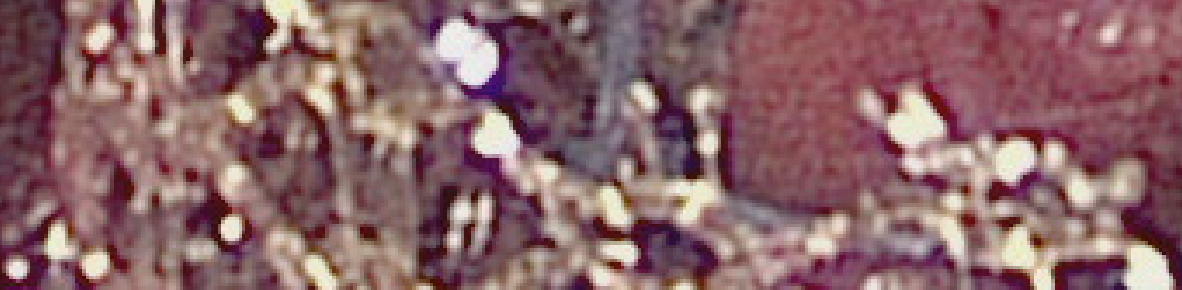

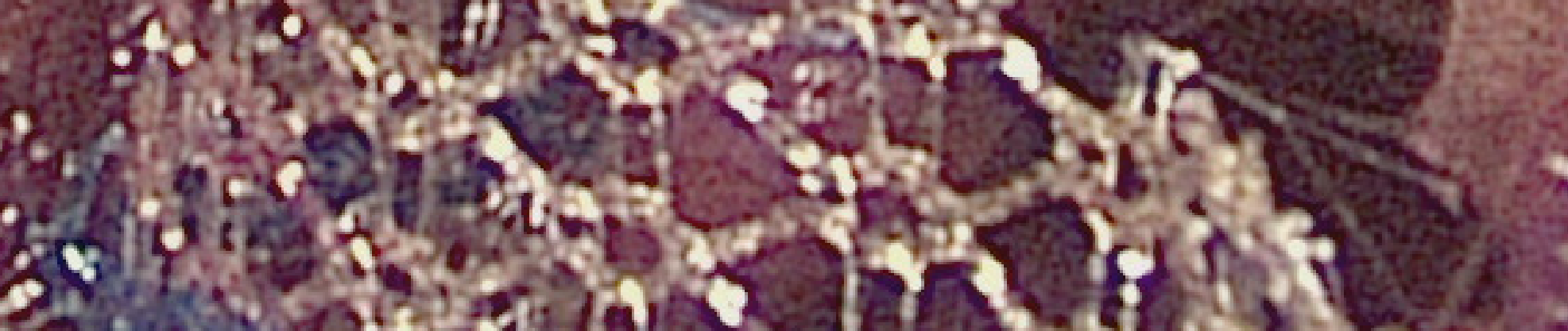

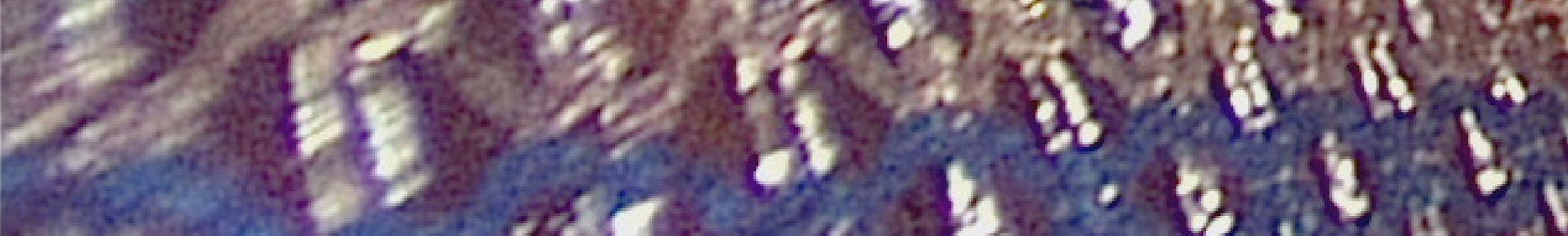




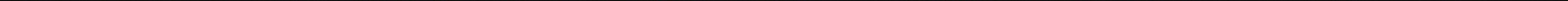




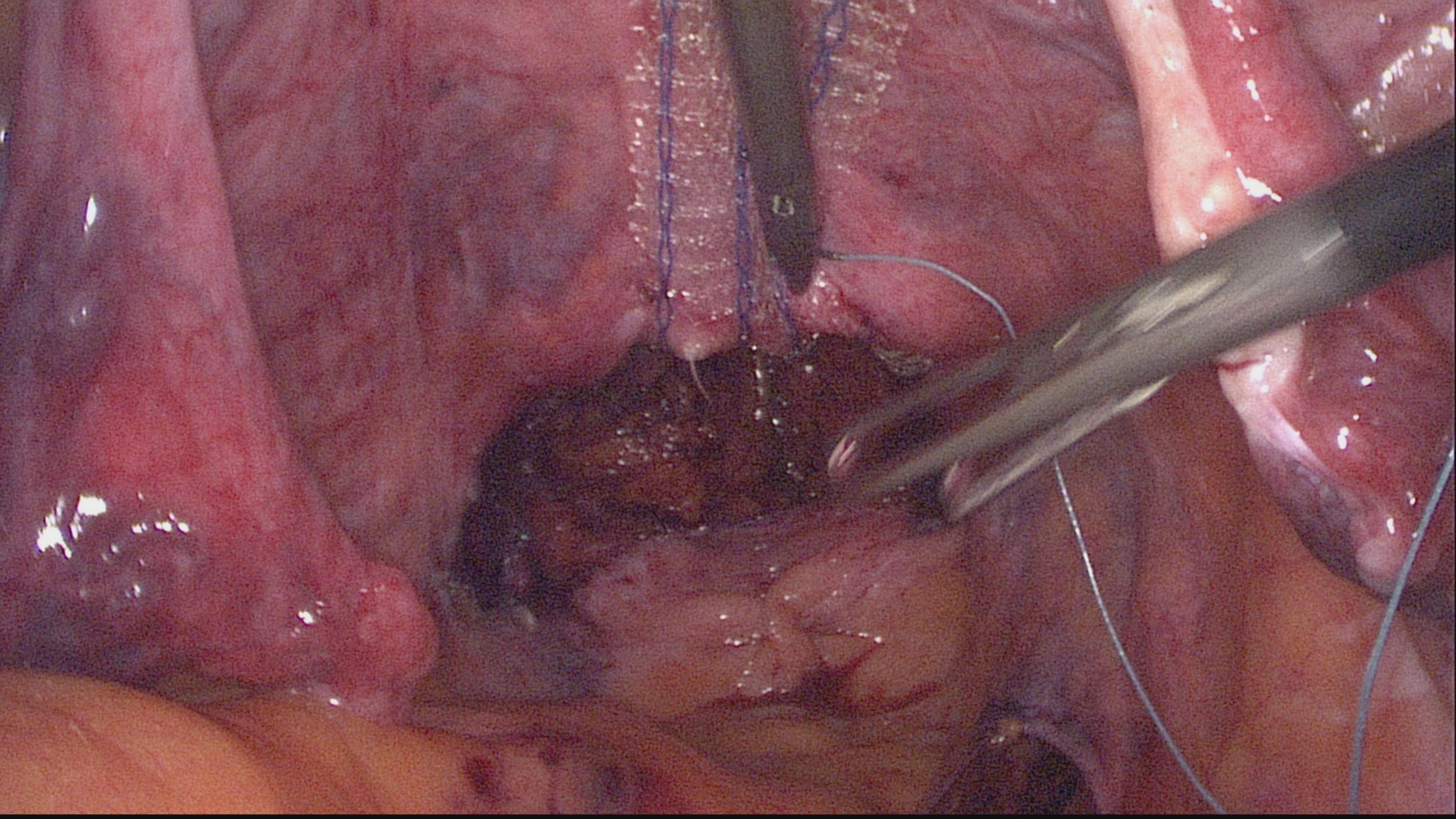




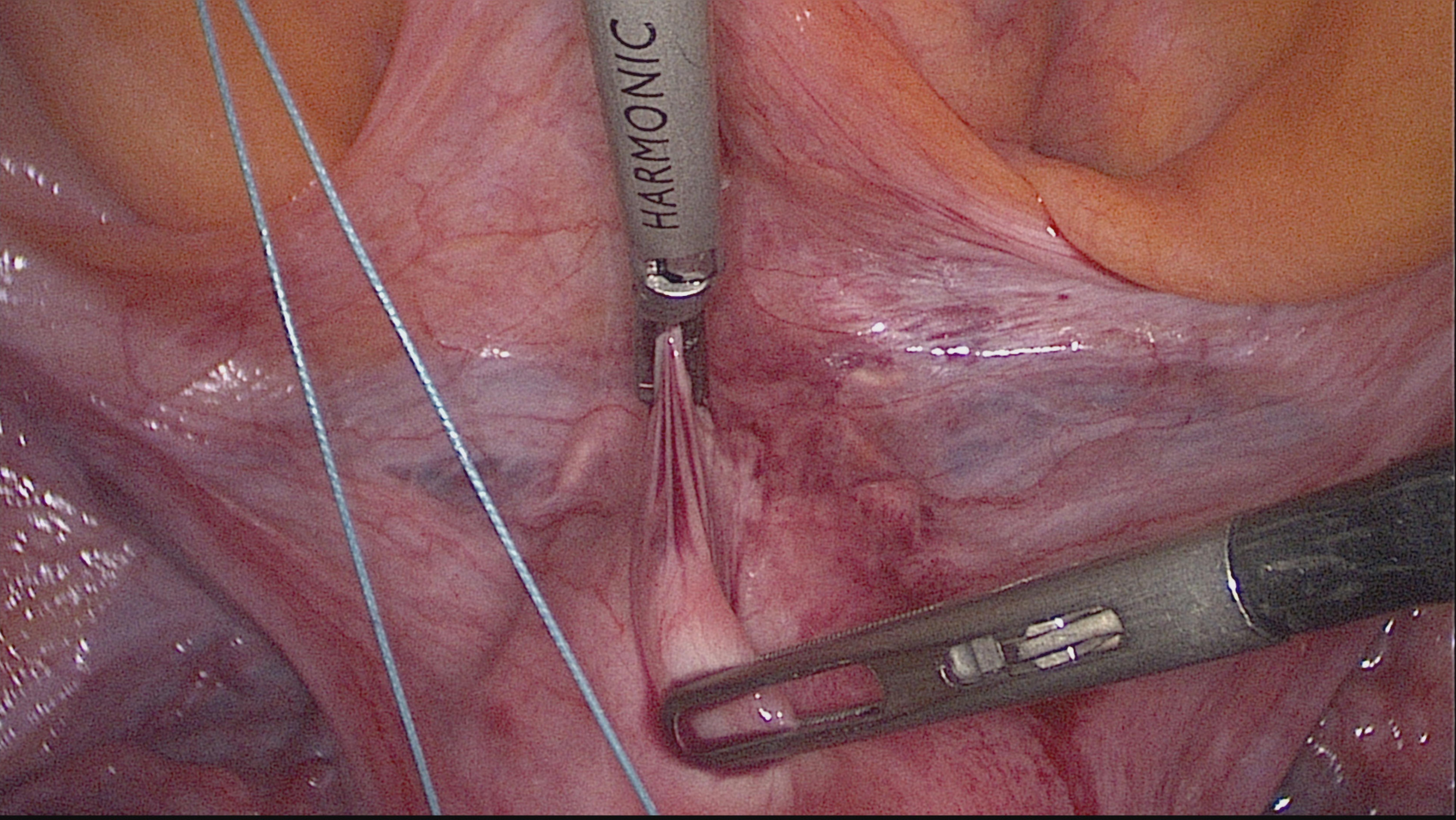




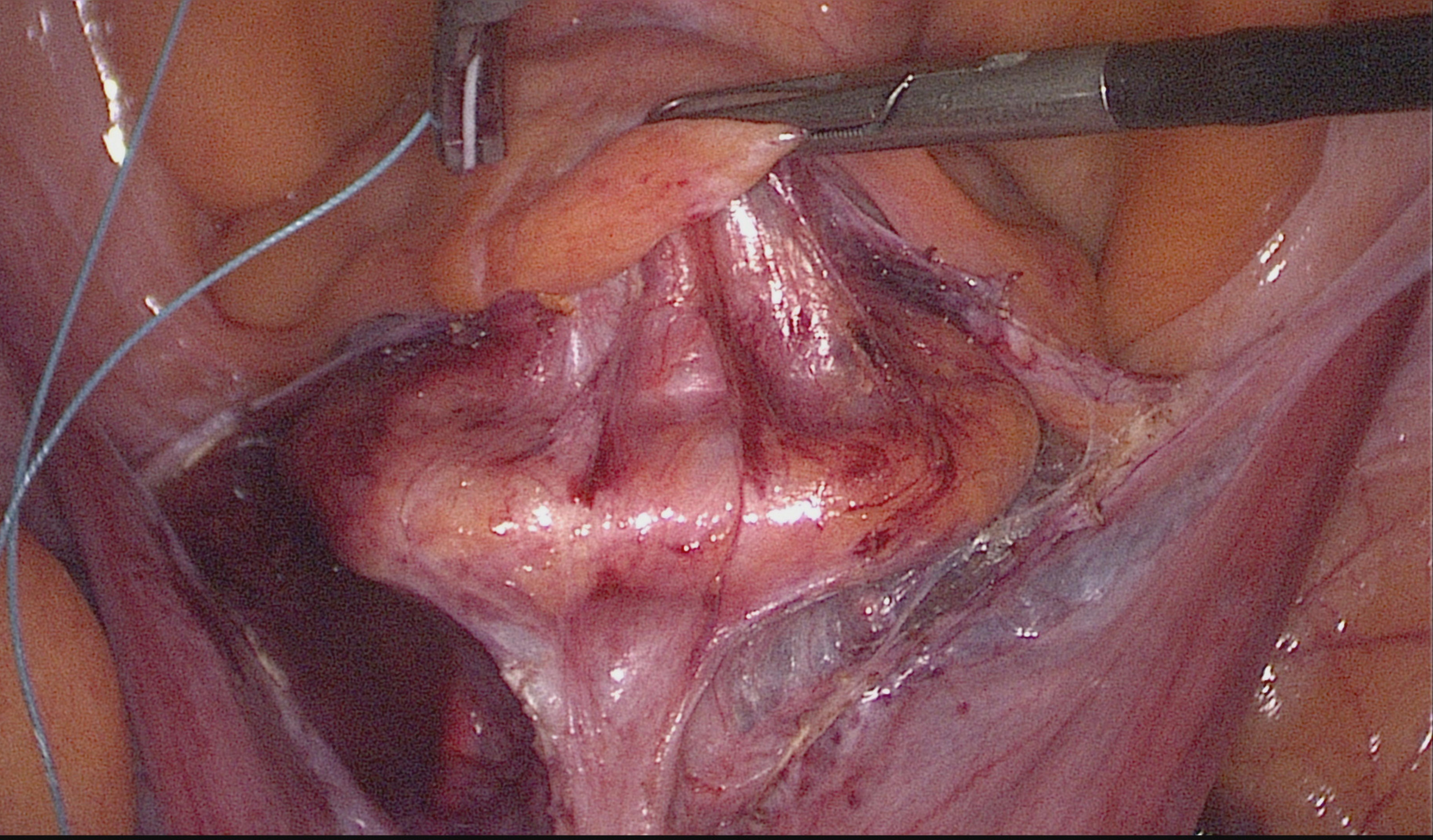






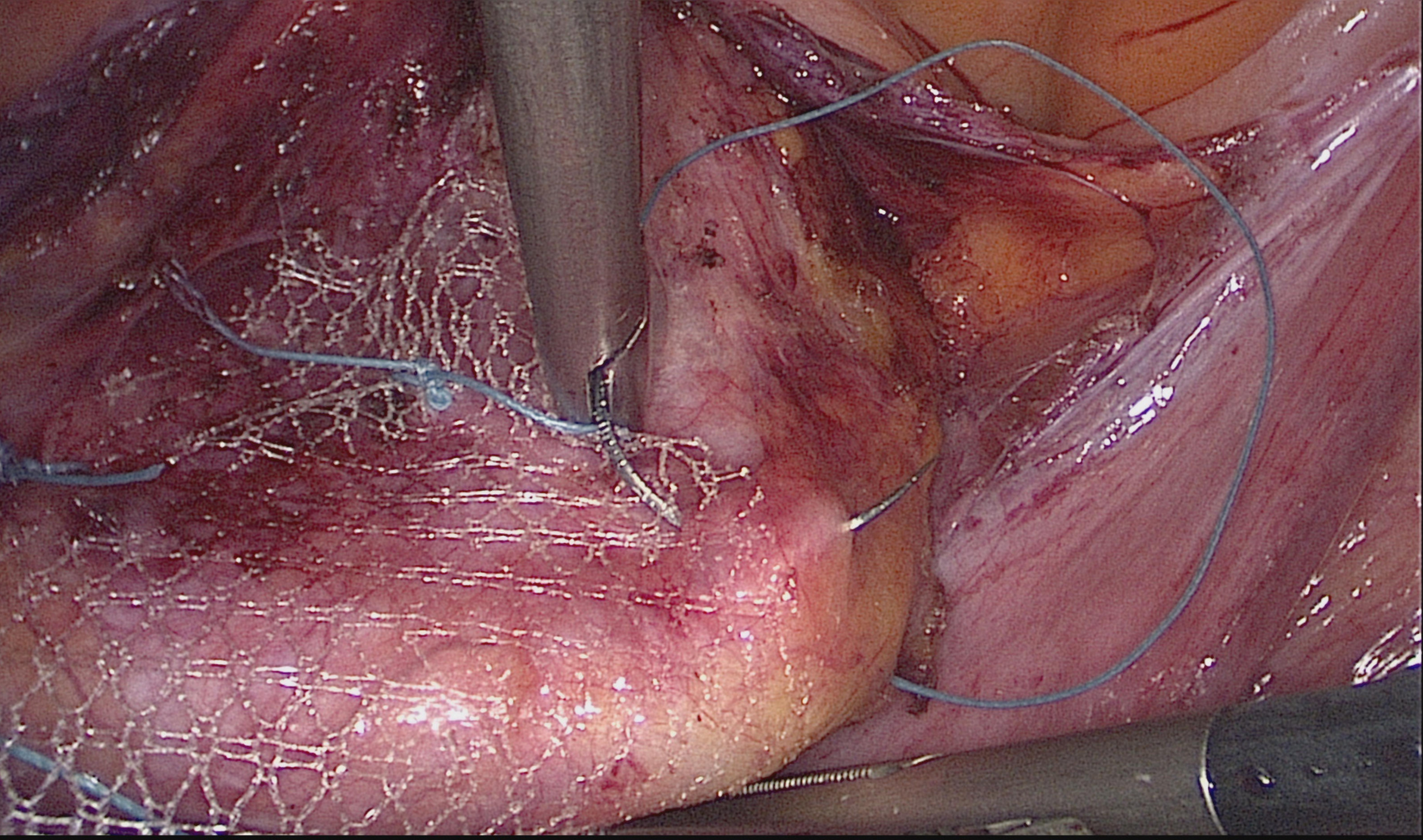




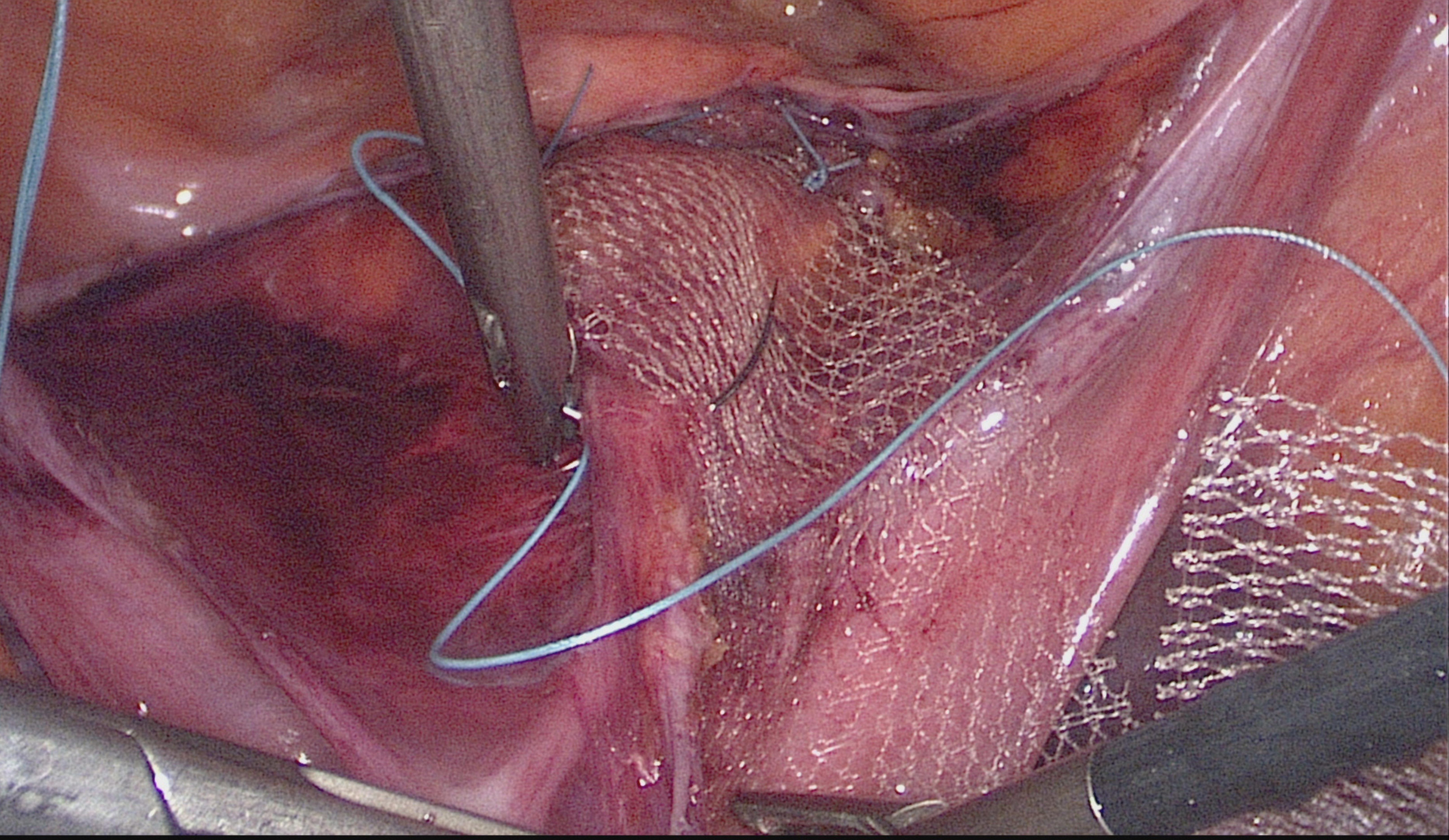




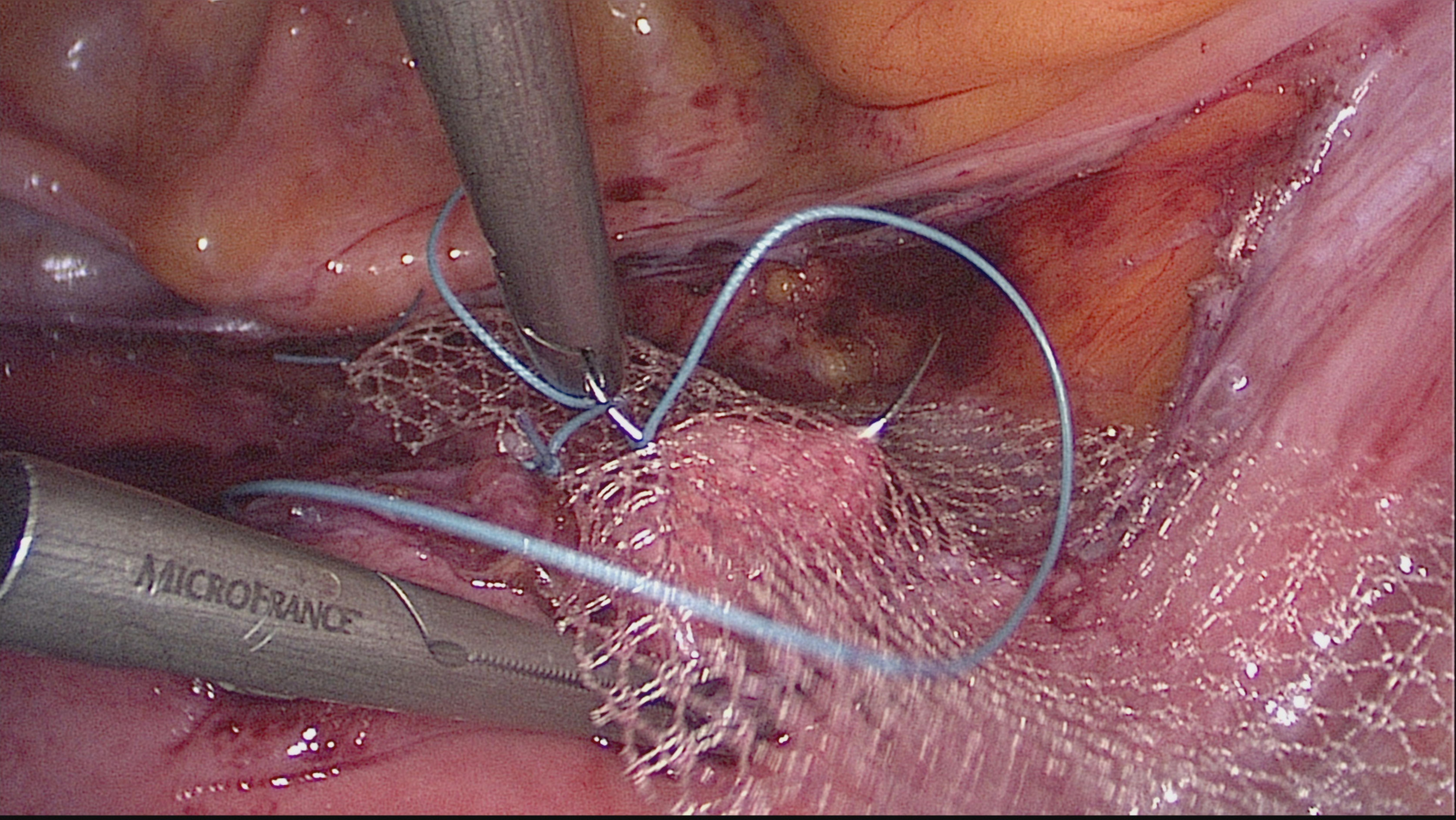




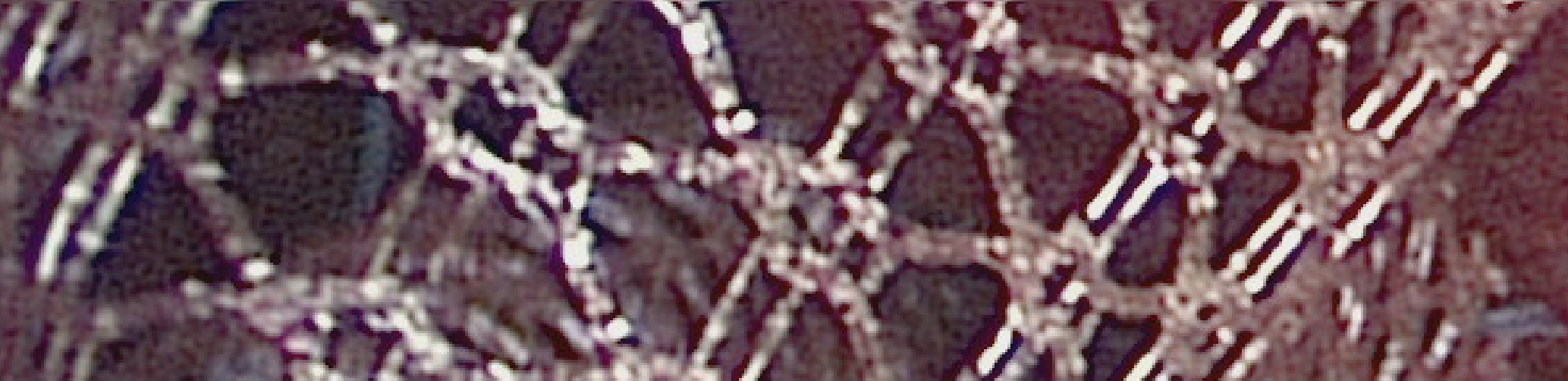

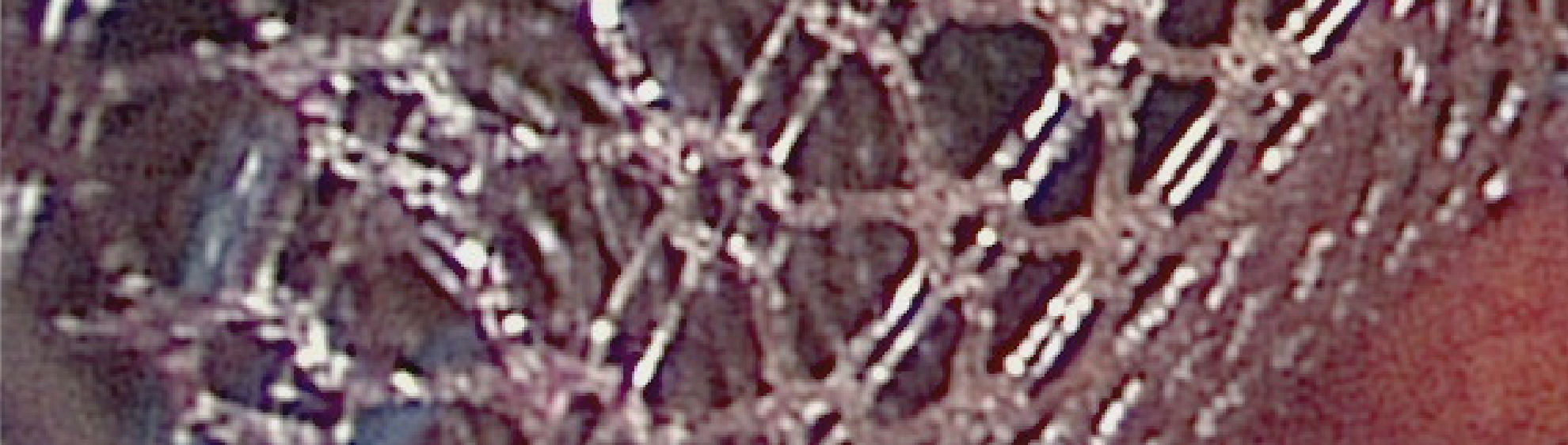

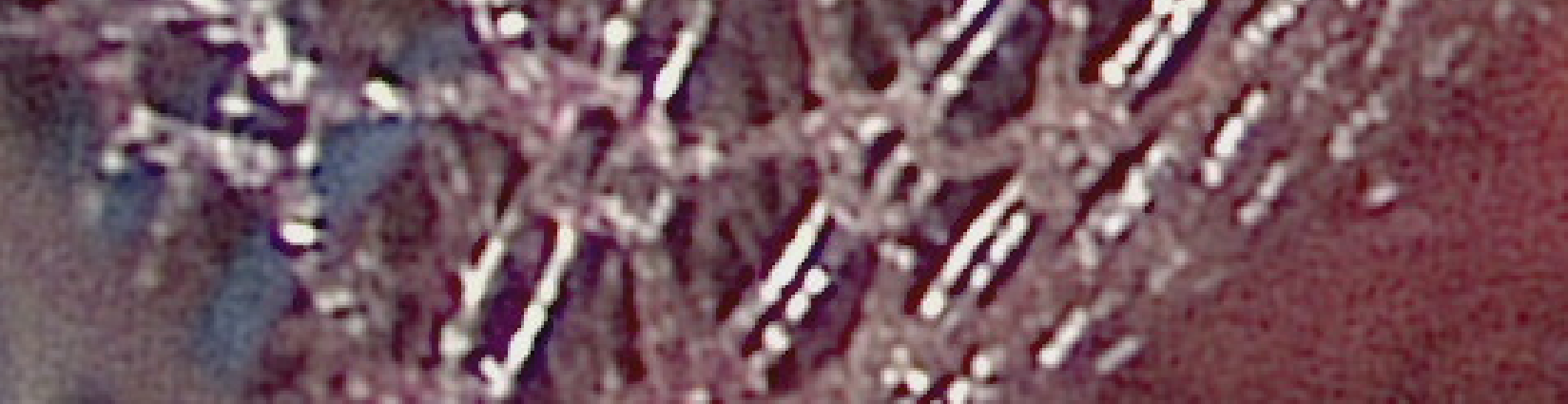

(2) 19

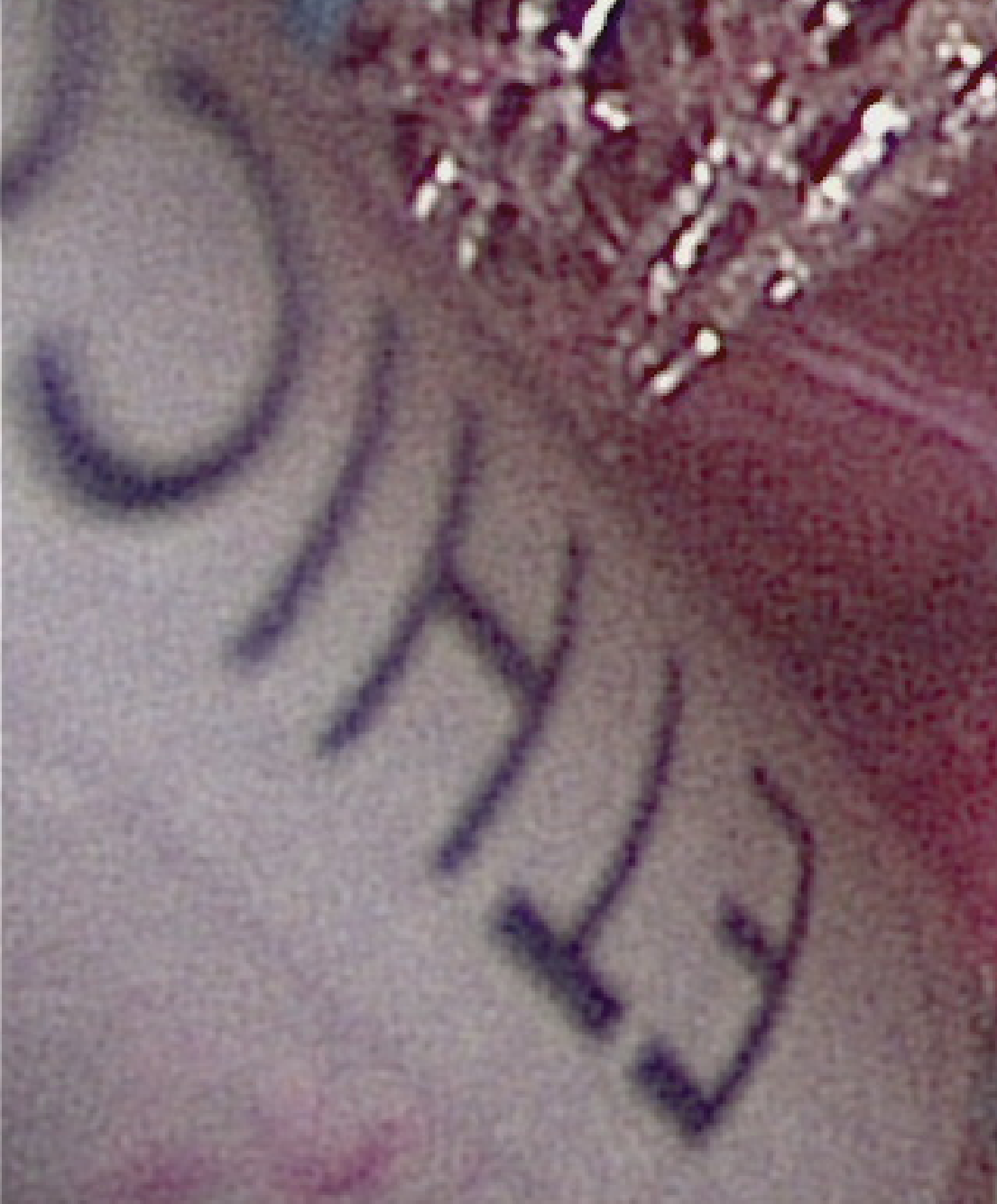

()
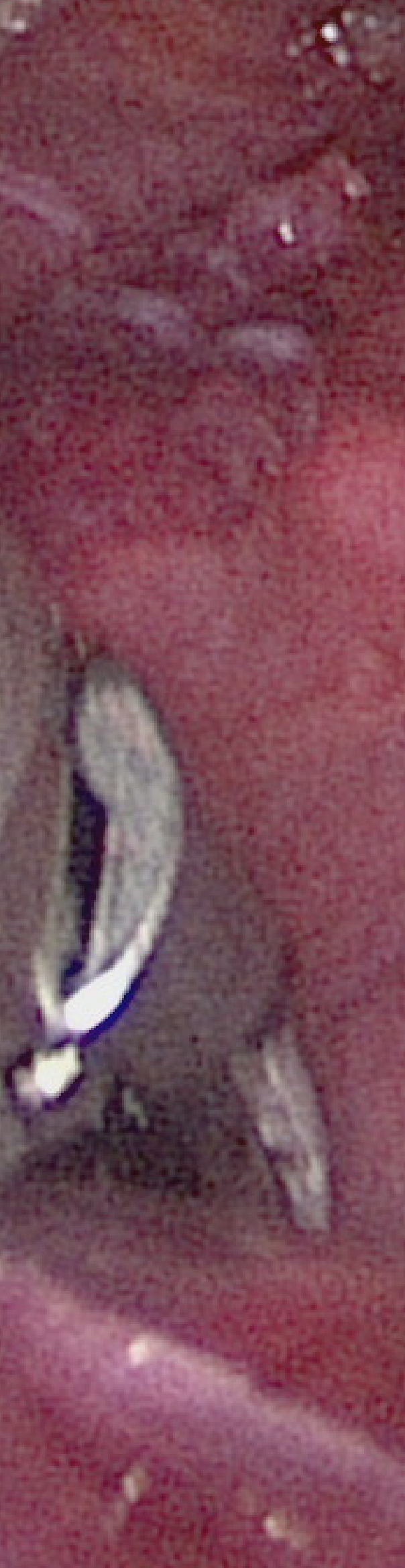

(-. $x^{2}: 5$
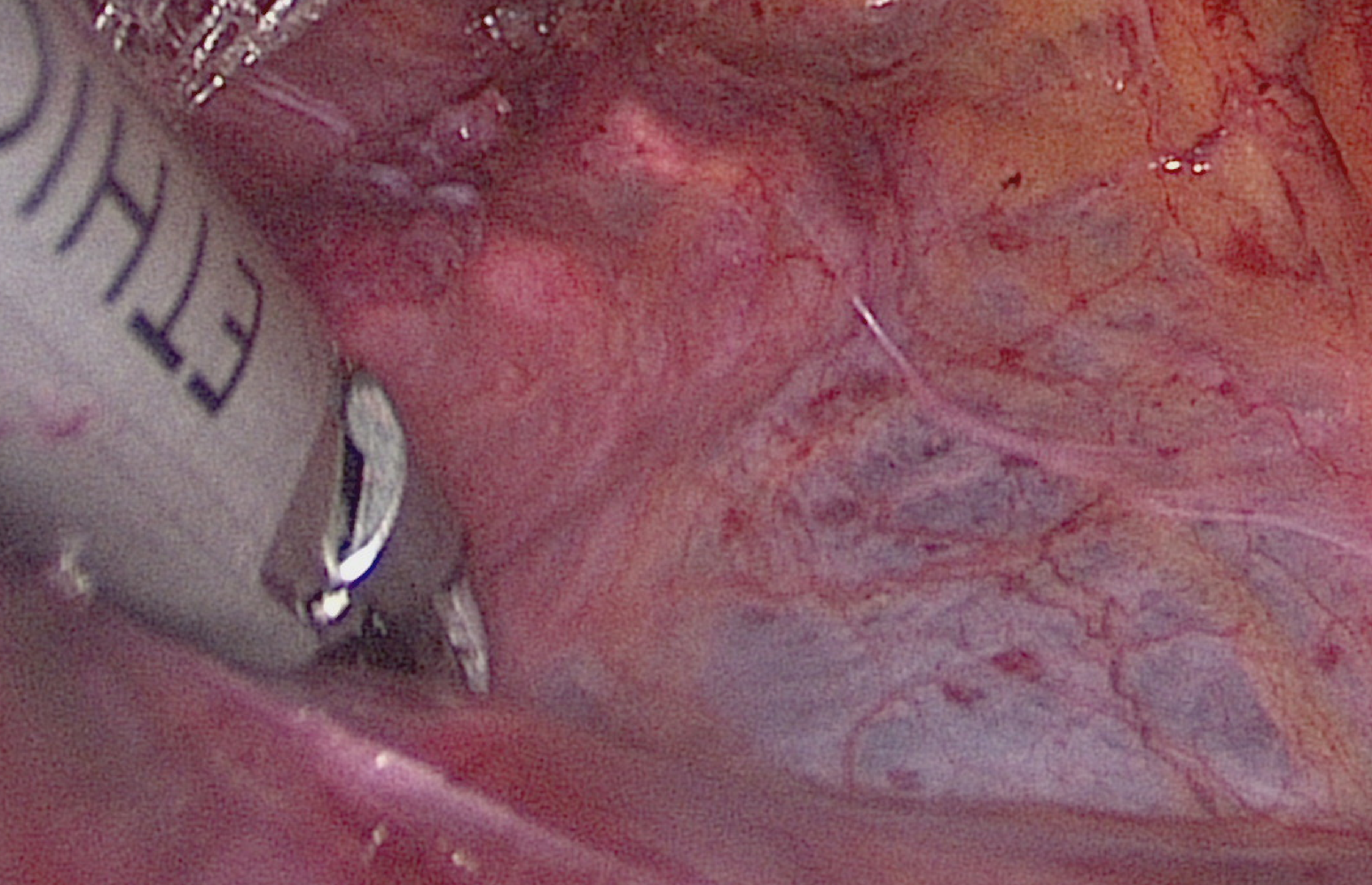

(i)

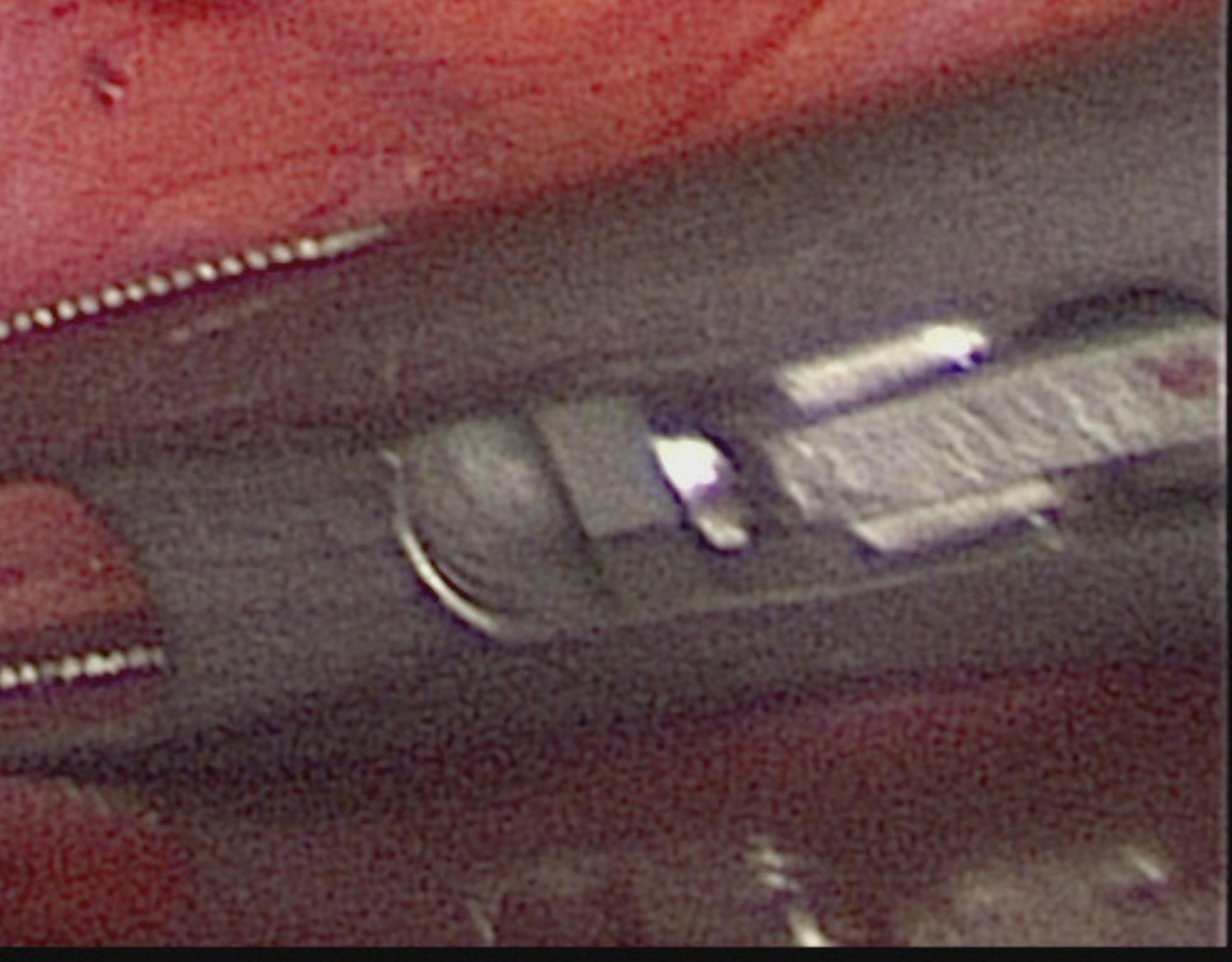




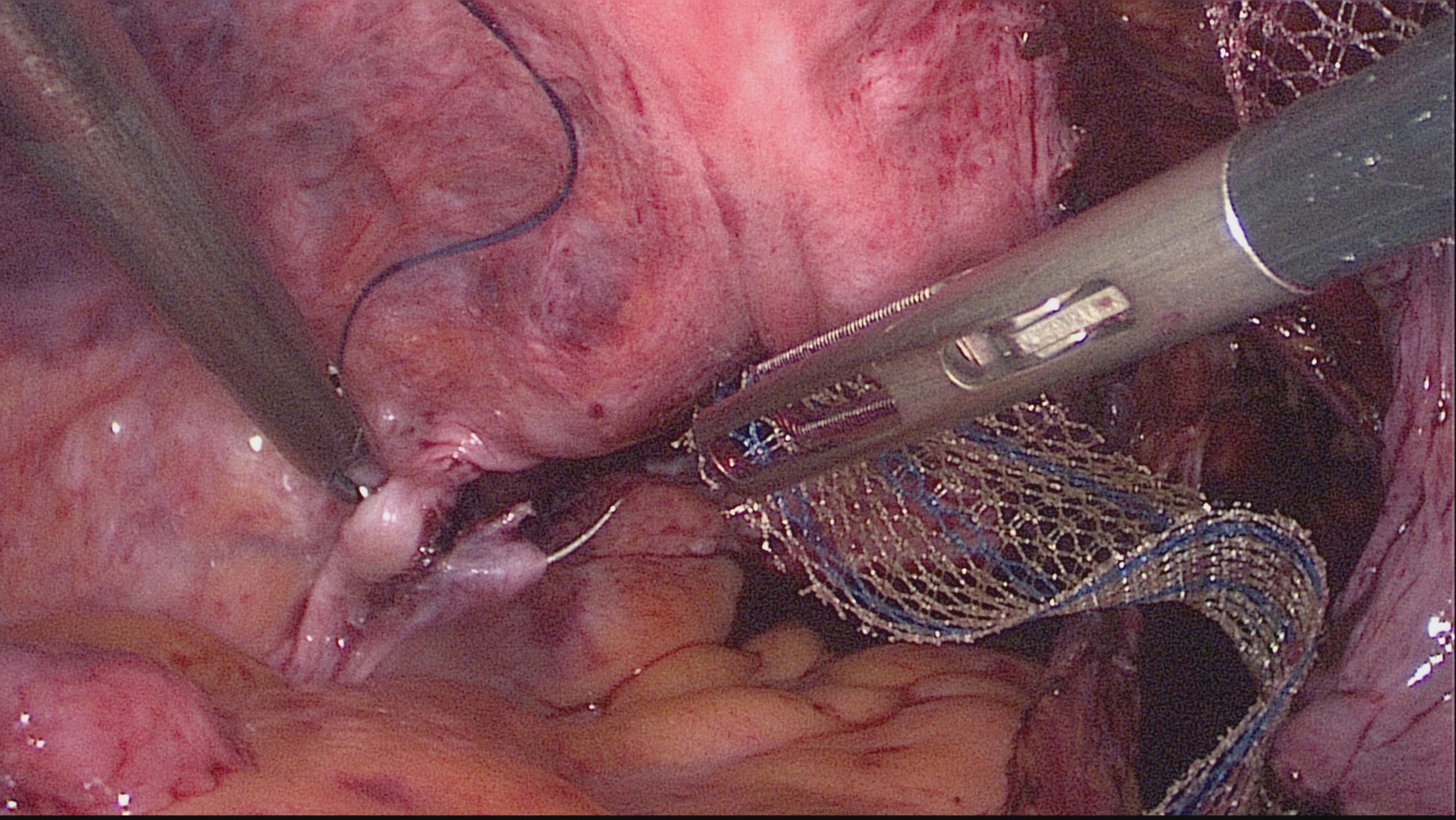




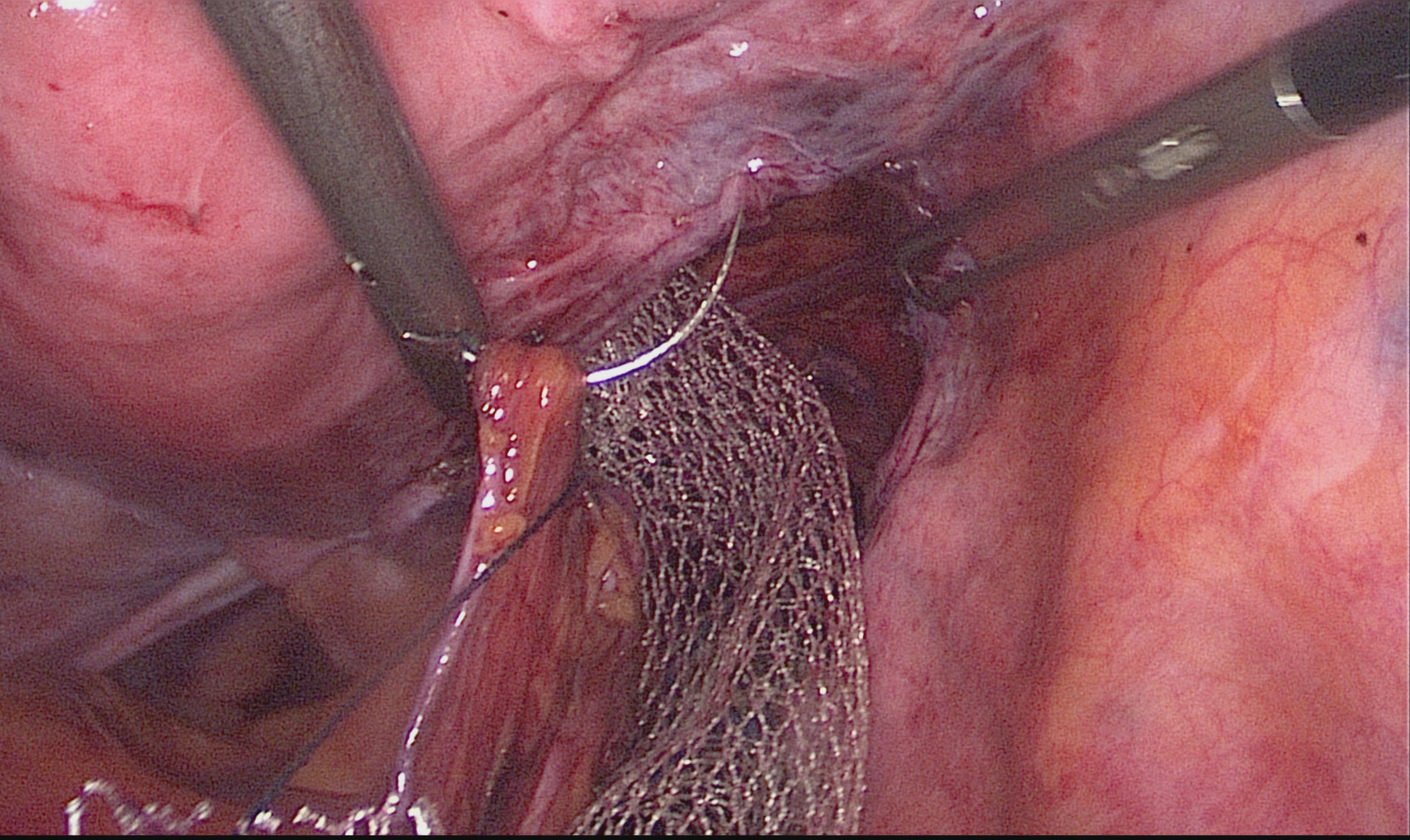




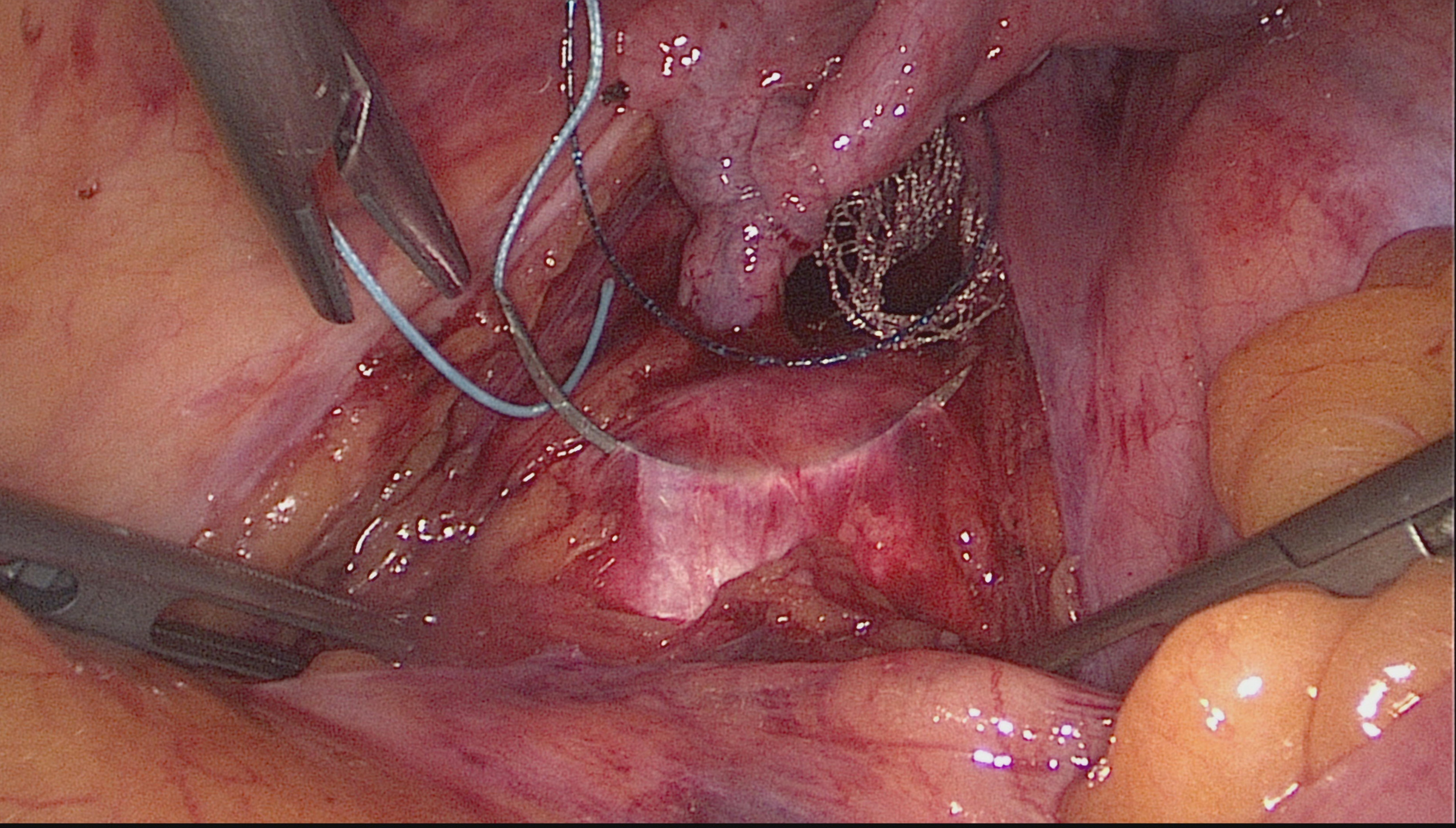




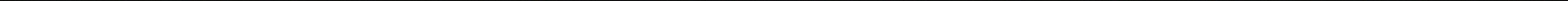

